\title{
Vortex Methods. I: Convergence in Three Dimensions
}

\author{
By J. Thomas Beale* and Andrew Majda**
}

\begin{abstract}
Recently several different approaches have been developed for the simulation of three-dimensional incompressible fluid flows using vortex methods. Some versions use detailed tracking of vortex filament structures and often local curvatures of these filaments, while other methods require only crude information, such as the vortex blobs of the two-dimensional case. Can such "crude" algorithms accurately account for vortex stretching and converge? We answer this question affirmatively by constructing a new class of "crude" three-dimensional vortex methods and then proving that these methods are stable and convergent, and can even have arbitrarily high order accuracy without being more expensive than other "crude" versions of the vortex algorithm.
\end{abstract}

1. Introduction. Vortex methods have provided an attractive and successful approach for the numerical simulation of incompressible fluid flow at high Reynolds number in two dimensions. They have several distinctive features: (1) the interactions of the computational vortices mimic the physical mechanisms in actual fluid flow; (2) vortex methods are automatically adaptive, since the vortex "blobs" concentrate in the regions of physical interest; and (3) there are no inherent errors which behave like the numerical viscosity of conventional Eulerian difference methods. Such diffusive errors can swamp the effects of physical viscosity in high Reynolds number flow similation.

The earliest attempts to represent flows by a vortex method used point vortices, and thus permitted arbitrarily large local velocities (Rosenhead [20]). Chorin [3] and Kuwahara and Takami [24] introduced the idea of using a finite vortex core, rather than a point vortex, to stabilize the method. Subsequently there have been a large number of successful two-dimensional flow simulations using an approximation of this type. (See the excellent recent survey by Leonard [16].) The actual fluid can undergo substantial distortion, while the computational vortex blobs do not distort at all. Thus the theoretical analysis by Hald [10] for 2-D inviscid flows is especially important, since he proves that a particular class of $2-\mathrm{D}$ vortex methods converge with second order accuracy for arbitrarily large time intervals.

There is now considerable interest in finding an appropriate formulation of the vortex method for three-dimensional fluid flows which retains the computational features (1)-(3) described above. This problem is the main subject of this paper. The

Received April 21, 1981; revised October 27, 1981.

1980 Mathematics Subject Classification. Primary 65M15, 76C05.

Key words and phrases. Vortex method, incompressible flow.

* Partially supported by N.S.F. Grant MCS-78-00908.

** Partially supported by N.S.F. Grant MCS-79-02735. 
design of vortex algorithms for three-dimensional flow is inherently more difficult than the two-dimensional case. In 2-D the vorticity is conserved along particle paths, whereas in 3-D the dynamics of vortex stretching is a prominent and complex phenomenon which must be reproduced by the method.

Several different versions of such three-dimensional vortex methods have been proposed and used in 3-D flow simulations by Leonard [16], [17]. [18], Del Prete [8], and Chorin [4], [5]. The methods of Leonard and Del Prete require a large amount of detailed information since entire vortex filament structures must be located and tracked, and often [8], [17] local curvatures of the vortex filaments are also computed. On the other hand, the three-dimensional vortex blob method recently introduced by Chorin [4], [5] is more flexible and requires less information. Rather than tracking entire vortex filament structures, it instead couples a vortex blob method with a local segment approximation which incorporates, in an Eulerian fashion, the local vortex stretching. Can such a "crude" three-dimensional vortex algorithm accurately represent fluid flows?

In the work presented here, we answer this question affirmatively. We formulate below a new class of three-dimensional vortex methods and then prove that these 3-D vortex methods are stable and convergent with arbitrarily high order accuracy. In these new algorithms, we update the velocity crudely in a fashion completely analogous to the 3-D vortex blob method of Chorin; however, unlike the algorithm in [4], we incorporate the vortex stretching through a Lagrangian update. This modification retains the desirable computational features in (1)-(3) above while again requiring only crude information - no vortex filaments need to be tracked as a whole, and no local curvature information is explicitly used. It is somewhat surprising that the methods introduced here are "crude", requiring roughly the same amount of computational labor as Chorin's algorithms, and yet are stable and highly accurate. Such methods can even have "infinite order" accuracy, i.e., a rate of convergence of order $h^{r}, r$ arbitrary, depending only on the smoothness of the underlying flow. In fact our techniques also yield stable, convergent vortex methods of arbitrary accuracy in 2-D and answer a variety of questions raised by Hald [10] and Leonard [16]. We postpone a detailed discussion of the 2-D algorithms until Part II of this work [1].

The Physical Equations and the 3-D Vortex Algorithm. We now describe the vorticity-stream formulation of the ideal incompressible fluid equations and introduce the class of three-dimensional vortex methods which we analyze here. Let $z=\left(z_{1}, z_{2}, z_{3}\right)$ denote the position, $u(z, t)=\left(u_{1}, u_{2}, u_{3}\right)$ the fluid velocity, and $\omega(z, t)=\left(\omega_{1}, \omega_{2}, \omega_{3}\right)=$ curl $u$ the vorticity. The trajectory $z(t ; \alpha)$ of a fluid particle starting at time 0 at the position $\alpha=\left(\alpha_{1}, \alpha_{2}, \alpha_{3}\right)$ is determined by the equation

$$
\frac{d z}{d t}=u(z, t), \quad z(0 ; \alpha)=\alpha
$$

We denote the solution of the ordinary differential equation (1.1) by $z(t ; \alpha)=\Phi^{t}(\alpha)$. Thus $\Phi^{t}$ is the transformation which describes the evolution of the fluid from time 0 to time $t$. We will write $\nabla_{\alpha} \Phi^{t}(\alpha)$ for the $3 \times 3$ Jacobian matrix $\partial \Phi_{i}^{t}(\alpha) / \partial \alpha_{j}, 1 \leqslant i$, $j \leqslant 3$. The velocity $u(z, t)$ is determined by the vorticity $\omega(z, t)$, since div $u=0$ and 
curl $u=\omega$. The expression for the velocity is the Biot-Savart law (e.g., [16, p. 307]),

$$
u(z, t)=\int_{\mathbf{R}^{3}} K\left(z-z^{\prime}\right) \omega\left(z^{\prime}, t\right) d z^{\prime},
$$

where $K$ is the matrix-valued kernel

$$
K(z)=-\frac{1}{4 \pi} \frac{z}{|z|^{3}} \times .
$$

The stretching of vorticity can be described by the familiar relation

$$
\omega_{t}+u \cdot \nabla \omega=\omega \cdot \nabla u,
$$

but it is more convenient here to use a Lagrangian formula due to Cauchy:

$$
\omega\left(\Phi^{t}(\alpha), t\right)=\nabla_{\alpha} \Phi^{t}(\alpha) \cdot \omega(\alpha, 0) .
$$

Thus the vorticity at the current location of a particle is the initial vorticity at the particle's original location, transformed by the Jacobian matrix of the flow. Derivations of (1.4) may be found, e.g., in [7, p. 32], [25, p. 84], or [26, p. 152]. After differentiating (1.4) with respect to $t$ and using (1.1), we arrive at the equation

$$
\frac{d}{d t} \omega\left(\Phi^{t}(\alpha), t\right)=\nabla_{\alpha} u\left(\Phi^{t}(\alpha), t\right) \cdot \omega_{0}(\alpha) .
$$

Here $\omega_{0}(\alpha)=\omega(\alpha, 0)$. The equations (1.1), (1.2), and (1.5) completely determine the motion of an inviscid incompressible fluid in three dimensions.

To describe the class of 3-D vortex methods which we analyze here, we introduce the lattice of integer-valued vectors in $\mathbf{R}^{3}$

$$
\Lambda=\left\{\left(z_{1}, z_{2}, z_{3}\right): z_{j} \in Z, j=1,2,3\right\}
$$

and the discrete grid $\Lambda^{h}=h \Lambda$ for $h>0$. We use the notation $z_{i}(t)=\Phi^{t}(i h)$, $u_{i}(t)=u\left(z_{i}(t), t\right), \omega_{i}(t)=\omega\left(z_{i}(t), t\right)$ to denote the position, velocity, and vorticity at time $t$ of a particle in the ideal flow beginning at a grid point $i h \in \Lambda^{h}$. We assume the initial vorticity has support inside some bounded set, say

$$
\Re_{0}=\left\{z:\left|z_{j}\right|<R_{0}, j=1,2,3\right\} .
$$

We denote by $\Lambda_{0}^{h}$ the intersection of $\Lambda^{h}$ and $\Re_{0}$. As in [4], and in complete analogy to the two-dimensional vortex method, we replace $K$ in (1.2) by a smoothed kernel $K_{\delta}$, where

$$
K_{\delta}(z)=\int K\left(z-z^{\prime}\right) \psi_{\delta}\left(z^{\prime}\right) d z^{\prime}
$$

and $\psi_{\delta}\left(z^{\prime}\right)=\delta^{-3} \psi\left(z^{\prime} / \delta\right)$. Here $\psi$ is a scalar cutoff function satisfying $\int \psi d z^{\prime}=1$ and other stability and accuracy conditions which we state precisely in Section 2. The choice of $\delta$ is discussed below. We will also approximate the gradient $\left(\partial / \partial \alpha_{1}, \partial / \partial \alpha_{2}, \partial / \partial \alpha_{3}\right)$ in (1.4) by some standard consistent finite difference operator $\nabla_{\alpha}^{h}$ on the lattice $\Lambda^{h}$.

We can now describe the strategy of the 3-D vortex algorithm. Given $h>0$, we compute $\tilde{z}_{i}(t)$ and $\tilde{\omega}_{i}(t)$, for $i h \in \Lambda_{0}^{h}$, as solutions of the coupled nonlinear ordinary differential equations

$$
\frac{d \tilde{z}_{i}}{d t}=\tilde{u}_{i}^{h}(t), \quad \tilde{z}_{i}(0)=i h,
$$




$$
\frac{d \tilde{\omega}_{i}}{d t}=\nabla_{\alpha}^{h} \tilde{u}_{i}^{h}(t) \cdot \omega_{0}(i h), \quad \tilde{\omega}_{i}(0)=\omega_{0}(i h) .
$$

The discrete velocity $\tilde{u}_{i}^{h}(t)$ is computed from $\tilde{z}_{j}$ and $\tilde{\omega}_{j}$ using the discrete Biot-Savart Law

$$
\tilde{u}_{i}^{h}(t)=\sum_{j h \in \Lambda_{0}^{h}} K_{\delta}\left(\tilde{z}_{i}(t)-\tilde{z}_{j}(t)\right) \tilde{\omega}_{j}(t) h^{3} .
$$

Obviously (1.7)-(1.9) are approximations to the equations (1.1), (1.2), (1.5) of the ideal fluid flow. Thus (1.7) computes approximate paths for a discrete set of particles, and (1.8) gives the vorticity of the particles.

In (1.8), $\nabla_{\alpha}^{h} \tilde{u}_{i}^{h}(t)$ needs to be computed only at points $i h$ in the support of $\omega_{0}$. Therefore the set of points where (1.7) and (1.8) are integrated, and (1.9) is evaluated, need only by slightly larger than the set of points $\Lambda_{0}^{h} \cap \operatorname{supp} \omega_{0}$. We have used the full set $\Lambda_{0}^{h}$ only for notational convenience.

The continuous velocity field defined by this vortex method is the natural interpolation of (1.9) given by

$$
\tilde{u}^{h}(z, t)=\sum_{j h \in \Lambda_{0}^{h}} K_{\delta}\left(z-\tilde{z}_{j}(t)\right) \tilde{\omega}_{j}(t) h^{3} .
$$

The most important property of this method is that $\tilde{u}^{h}$ accurately approximates the exact velocity field (see Theorem A below). We will denote by $u^{h}$ and $u_{i}^{h}$ the quantities defined as in (1.10) and (1.9) from the discrete Biot-Savart Law, but evaluated with the particle positions and vorticities of the actual flow:

$$
\begin{aligned}
& u^{h}(z, t)=\sum_{j h \in \Lambda_{0}^{h}} K_{\delta}\left(z-z_{j}(t)\right) \omega_{j}(t) h^{3}, \\
& u_{i}^{h}(t)=\sum_{j h \in \Lambda_{0}^{h}} K_{\delta}\left(z_{i}(t)-z_{j}(t)\right) \omega_{j}(t) h^{3} .
\end{aligned}
$$

Statement of Convergence Results in a Special Case. The stability and accuracy of the three-dimensional vortex method defined in (1.7)-(1.9) above is controlled by the properties of the cutoff $\psi$, the difference operator $\nabla_{\alpha}^{h}$, and the choice $\delta=O\left(h^{q}\right)$, $0<q<1$. We illustrate the theory developed in Sections $2-5$ by stating the results with specific choices which allow arbitrarily high order accuracy. The theorem stated below is a special case of Theorem 1 in Section 2.

For any positive integer $p$, we choose for $\psi$ the function $\psi^{(2 p)}$, which is the inverse Fourier transform of the generalized Gaussian, $\exp \left(-|\xi|^{2 p}\right)$; i.e.,

$$
\psi^{(2 p)}(z)=c_{p} \int_{\mathbf{R}^{3}} e^{2 \pi i z \cdot \xi} e^{-|\xi|^{2 p}} d \xi
$$

with $c_{p}$ chosen so that $\psi^{(2 p)}$ has integral 1. In practice $\psi^{(2 p)}(z)$ can be computed to arbitrary accuracy by one-dimensional quadrature; for a radially symmetric function $g(z)$ [21, p. 155],

$$
g(z)=2 \pi|z|^{-1 / 2} \int_{0}^{\infty} \hat{g}(|\xi|) J_{1 / 2}(2 \pi|z||\xi|)|\xi|^{3 / 2} d|\xi|,
$$

where $J_{1 / 2}$ is the Bessel function of order $\frac{1}{2}$. (See [16] for a similar choice in 2-D. Smoothed cutoffs of the type used in [10] are also possible.) 
We choose for $\nabla_{\alpha}^{h}$ the standard $2 p$-order accurate antisymmetric centered difference operator $\nabla_{\alpha}^{h, 2 p}$, where $\nabla_{\alpha}^{h, 2 p}=\left(D_{\alpha_{1}}^{h, 2 p}, D_{\alpha_{2}}^{h, 2 p}, D_{\alpha_{3}}^{h, 2 p}\right)$ and

$$
D_{\alpha_{j}}^{h, 2 p}=\sum_{\nu=1}^{p} \gamma_{\nu} D_{\alpha_{j}}^{0}(\nu h)
$$

and

$$
\gamma_{\nu}=\frac{-2(-1)^{\nu}(p !)^{2}}{(p+\nu) !(p-\nu) !}
$$

(see [14]). Here $D_{\alpha_{j}}^{0}(l)$ is the usual centered difference quotient scaled to a grid of length $l$ in the $j$ th coordinate direction.

We write $|\cdot|_{0}$ for the continuous $L^{2}$ (or $H^{0}$ ) norm of a function and $|\cdot|_{0, S}$ for the norm restricted to a set $S$. We also use the notation $\left|u_{i}\right|_{0, h}$ for the discrete $H^{0}$ norm on $\Lambda_{0}^{h}\left(\right.$ or $\left.\Lambda^{h}\right)$ defined by

$$
\left|u_{i}\right|_{0, h}^{2}=\sum_{i h \in \Lambda_{0}^{h}}\left|u_{i}\right|^{2} h^{3}
$$

with corresponding inner product

$$
\left(u_{i}, v_{i}\right)_{h}=\sum_{i h \in \Lambda_{0}^{h}} u_{i} v_{i} h^{3} .
$$

(It should always be clear from the context whether the norm is restricted to $\Lambda_{0}^{h}$.)

The following theorem asserts that the vortex method introduced above is convergent with accuracy of order $2 p$, provided $\psi=\psi^{(2 p)}$ and $\nabla_{\alpha}^{h}=\nabla_{\alpha}^{h, 2 p}$.

TheOREM A. Assume that the velocity field $u(z, t)$ is smooth (has sufficiently many derivatives in $L^{2}$ ) for $0 \leqslant t \leqslant T$ and that the initial vorticity has bounded support. Let $p \geqslant 2$ be a positive integer, and let $\varepsilon>0$ be arbitrarily small but fixed.

Suppose that we compute $\tilde{z}_{i}$ and $\tilde{\omega}_{i}$, ih $\in \Lambda_{0}^{h}$, according to the algorithm (1.7)-(1.9), with $\delta=h^{1-\varepsilon}, \psi$ the cutoff $\psi^{(2 p)}$ of (1.13), and $\nabla_{\alpha}^{h}$ the difference operator $\nabla_{\alpha}^{h, 2 p}$ of (1.14). Then for $h \leqslant h_{0}$ we have for this algorithm

(1) Convergence of the particle paths

$$
\max _{0 \leqslant t \leqslant T}\left|\tilde{z}_{i}(t)-z_{i}(t)\right|_{0, h} \leqslant C h^{2 p(1-\varepsilon)},
$$

(2) Convergence of the discrete velocity

$$
\max _{0 \leqslant t \leqslant T}\left|\tilde{u}_{i}^{h}(t)-u_{i}(t)\right|_{0, h} \leqslant C h^{2 p(1-\varepsilon)},
$$

(3) Convergence of the continuous velocity

$$
\max _{0 \leqslant t \leqslant T}\left|\tilde{u}^{h}(\cdot, t)-u(\cdot, t)\right|_{L^{2}\left(B\left(R_{0}\right)\right)} \leqslant C h^{2 p(1-\varepsilon)},
$$

for any bounded set $B\left(R_{0}\right)=\left\{z:|z|<R_{0}\right\}$. The constants depend on $T, p$, bounds for a finite number of derivatives of $u(z, t)$, the radius of support of $\omega_{0}$, and $R_{0}$. (The dependence on $R_{0}$ can be removed by a longer argument than the one below. The case $p=1$ can be included; see the remarks in Section 2 following Theorems 1 and 2.)

As mathematical background, we remind the reader of the existence theory for solutions of the equations of ideal fluid flow in $\mathbf{R}^{3}$, as described in the work of Kato 
[13] and Temam [22]. In [13] the existence of a classical solution of the Euler equations is shown for a time interval depending on the initial data. The main result of [22] shows that, if the initial data has a large number of derivatives in $L^{2}$, the solution has the same number of derivatives on the time interval determined in [13]; the time interval is independent of the number of derivatives. It follows from these results that, if $T$ in Theorem $\mathrm{A}$ is determined as in [13], the dependence of the constants on the solution reduces to dependence only on the initial data.

The Technical Features of the Proof. We discuss briefly the proof of the convergence statements in Theorem A above and the more general Theorem 1 of Section 2. In outline, the proof is patterned after the work of Hald [10] for two-dimensional flows-we show that consistency and stability imply convergence. However, quite different ideas from those in [10] are needed for both the stability and the consistency, partly because we have to assess the error in the vorticity as well as the positions of the particles, but also because sharper accuracy is required beyond that in [10] for a convergence proof in 3-D.

Since the discrete velocity is measured in $H_{h}^{0}$ and the vorticity is a first derivative of the velocity, it is natural to measure the vorticity in a discrete negatively normed Sobolev space, $H_{h}^{-1}$. To define $H_{h}^{-1}$, we recall that $H_{h}^{1}$ is the set of lattice functions $\left\{f_{i}\right\}$, ih $\in \Lambda^{h}$, with the norm

$$
\left|f_{i}\right|_{1, h}^{2}=\left|f_{i}\right|_{0, h}^{2}+\sum_{j=1}^{3}\left|D_{j}^{+} f_{i}\right|_{0, h}^{2},
$$

where $D_{j}^{+}$is the standard forward divided difference operator on the lattice $\Lambda^{h}$ along the $j$ th coordinate direction (see [23, Section I. 3.3]). As in the continuous case, we define $H_{h}^{-1}$ as the dual of $H_{h}^{1}$ with respect to the $H_{h}^{0}$ inner product, i.e., $w_{i} \in H_{h}^{-1}$ provided the discrete norm defined by

$$
\left|w_{i}\right|_{-1, h} \equiv \sup _{\left\{f_{i}\right\} \in H_{h}^{1}} \frac{\left|\left(w_{i}, f_{i}\right)_{h}\right|}{\left|f_{i}\right|_{1, h}}
$$

is finite. Since the algorithm in (1.7) and (1.8) involves a coupling of the particle paths and vortex stretching, we need to assess both effects simultaneously. In fact, under the hypothesis of Theorem A or Theorem 1, we prove the basic estimate

$$
\max _{0 \leqslant t \leqslant T}\left(\left|\tilde{z}_{i}-z_{i}\right|_{0, h}+\left|\tilde{\omega}_{i}-\omega_{i}\right|_{-1, h}\right) \leqslant C h^{2 p(1-\varepsilon)} .
$$

It is an essential aspect of the nonlinear stability proof that the errors are related more directly to integral operators than in the stability argument of [10]. As a consequence, we use only discrete $L^{1}$ estimates for the kernels. The reader can check that the proof in [10], which uses both $L^{1}$ and $L^{2}$ estimates for the kernels, could not be extended directly to the 3-D case because of the increased singularity of the kernel. Another novel feature is that we use discrete integral operators analogous to (1.9) as mappings from $H_{h}^{-1}$ to $H_{h}^{0}$ to measure the errors in velocity arising from errors in the vorticity.

In Section 2 we state the general convergence theorems and prove them, assuming the main stability and consistency estimates. The remainder of this paper is devoted to the proof of stability. After several technical lemmas are stated and discussed in Section 3, the main stability argument is given in full detail in Section 4. The lemmas 
of Section 3 are proved in Section 5. We postpone until Part II the detailed proof of the consistency lemma, used in the convergence argument of Section 2. The consistency uses ideas completely different from those developed here and in [10], and also has other applications which we treat in Part II. However, we state the consistency lemma in detail in Section 2, so that this paper can be read independently from Part II.

"Infinite Order" Accurate Vortex Methods. Finally, to illustrate the flexibility of the 3-D vortex algorithms defined in (1.7)-(1.9), we end this introduction by describing a class of "infinite order" vortex methods, i.e., algorithms having computed solutions which converge to the exact solution at a rate $O\left(h^{r}\right)$ for any $r>0$, provided the underlying fluid flow is $C^{\infty}$. Certain spectral approximations for periodic flow are the only other nonlinear algorithms for fluid flow which are known to have this rapid rate of convergence [11], [15].

We define a cutoff $\psi^{\infty}$ as the inverse Fourier transform of a smooth, rapidly decreasing function $\hat{\psi}^{\infty}(|\xi|)$ which is identically one for $|\xi|$ less than some fixed radius. (It is easy to build such functions explicitly; e.g., see [19, pp. 1074-1075] for a discussion and graph of a typical $\hat{\psi}^{\infty}$.) Let $\rho(z)$ be a fixed smooth function so that supp $\rho \subseteq \mathcal{R}_{0}$ and $\rho \equiv 1$ on the support of the initial vorticity $\omega_{0}$. Also let $\nabla_{\alpha}^{h, \infty}$ denote the spectral approximation to $\nabla_{\alpha}$, which can be implemented by the fast Fourier transform (see [15]), where any grid function with support inside $\Lambda_{0}^{h}$ can be identified with a discrete periodic function. Replace the vorticity correction (1.8) with

$$
\frac{\partial \tilde{\omega}_{i}}{\partial t}=\nabla_{\alpha}^{h, \infty}\left(\rho(i h) \tilde{u}_{i}^{h}\right) \cdot \omega_{0}(i h), \quad \tilde{\omega}_{i}(0)=\omega_{0}(i h), \quad i h \in \Lambda_{0}^{h}
$$

Then in analogy with Theorem A above, we have the following.

THEOREM B. Assume that the underlying fluid flow is $C^{\infty}$ until time T. Compute $\tilde{z}_{i}$ and $\tilde{\omega}_{i}$ by using the cutoff $\psi^{\infty}$ in (1.6) and the equation (1.18) in place of (1.8). Choose $\delta=h^{q}$ with $0<q<1$. Then the errors in (1)-(3) of Theorem A are estimated by $C_{r} h^{r}$ for any $r>0$ and some constant $C_{r}$, provided $h \leqslant h_{0}$.

M. Pearlman of University of California, Berkeley is currently testing the vortex algorithms we have described, and the results of these numerical experiments will be reported elsewhere at a later date. A further discussion of the practical utility of these methods is given in [2].

2. The Main Theorems. Before stating our main theorems, we give the precise hypotheses on the cutoff $\psi$ and the difference operator $\nabla_{\alpha}^{h}$ used in the Lagrangian vorticity update (1.8), which are needed for our main theoretical results. We remind the reader that the specific choices discussed in the introduction provide examples of the general theory developed below.

To describe the precise conditions on the cutoff $\psi$, we introduce the Fourier transform,

$$
\hat{\psi}(\xi)=\int e^{-2 \pi i z \cdot \xi} \psi(z) d z
$$


and recall that a function $\phi(z)$ is rapidly decreasing for $|z| \geqslant 1$ if $\phi$ is $C^{\infty}$ for $|z| \geqslant 1$, and for any integer $m>0$ and multi-index $\beta$,

$$
\sup _{|z| \geqslant 1}|z|^{m}\left|D_{z}^{\beta} \phi(z)\right| \leqslant C_{m, \beta}
$$

for some constant $C_{m, \beta}$. We also make the following definition:

Definition. We say the cutoff $\psi$ belongs to the class $F e S^{-M, p}$ provided the following conditions are satisfied:

(1) $\psi(z)$ belongs to $C^{2}\left(\mathbf{R}^{3}\right)$, and $\psi(z)$ is rapidly decreasing for $|z| \geqslant 1$.

(2) $\int \psi(z) d z=1, \int z^{\gamma} \psi(z) d z=0$, where $\gamma$ is any multi-index with $1 \leqslant|\gamma| \leqslant p-1$.

(3) $M$ is some positive number, and the Fourier transform $\hat{\psi}(\xi)$ satisfies, for any multi-index $\beta$ and some constant $C_{\beta}$,

$$
\sup _{\xi \in \mathbf{R}^{3}}\left|D_{\xi}^{\beta} \hat{\psi}(\xi)\right| \leqslant C_{\beta}(1+|\xi|)^{-M-|\beta|} .
$$

The need for these conditions will be apparent in the consistency argument; see the introduction to Part II. The notation $\mathrm{FeS}$ stands for the Fourier transform of a symbol class. We say that $\psi$ belongs to $F e S^{-\infty, p}$ provided $\hat{\psi}$ is rapidly decreasing, so that (3) is true for arbitrary $M>0$, and the condition in (2) is satisfied for a given $p$. In this case, condition (1) is automatically satisfied. We say that $\psi$ belongs to $\mathrm{FeS} \mathrm{S}^{-\infty, \infty}$ provided $\psi$ belongs to $\mathrm{FeS} \mathrm{S}^{-\infty, p}$ for any positive integer $p$. The cutoffs $\psi^{(2 p)}$ defined in (1.13) belong to $F e S^{-\infty, 2 p}$, while the cutoff described above (1.18) belongs to $F e S^{-\infty, \infty}$. The cutoffs $\psi$ belonging to $F e S^{-M, p}, M$ finite, are not smooth, and, as we shall see below, yield vortex methods with nonoptimal rates of convergence for smooth fluid flow when compared to cutoffs in $\mathrm{FeS}^{-\infty, p}$. (See the remarks following Theorems 1 and 2 for further discussion.)

Regarding the difference operator $\nabla_{\alpha}^{h}$, we impose a mild stability condition which is always satisfied for any standard finite difference approximation to $\nabla_{\alpha}$ such as the ones defined in (1.14), and also for the spectral derivative approximation in (1.18).

Stability for $\nabla_{\alpha}^{h}$. The operators $\nabla_{\alpha}^{h}$ are uniformly bounded as mappings from $H_{h}^{0}$ to $H_{h}^{-1}$. I.e., there exists a fixed constant $C$ so that, for $h \leqslant h_{0}$,

$$
\left|\nabla_{\alpha}^{h} v_{j}\right|_{-1, h} \leqslant C\left|v_{j}\right|_{0, h} .
$$

Given a multi-index $l$, we let $T^{l}$ denote grid translation in the direction $l$, i.e., $T^{l} w_{j}=w_{j-l}$. We have the following fact:

Proposition 2.1. (1) Every difference operator having the form

$$
\nabla_{\alpha}^{h}=\frac{1}{h} \sum_{|\eta| I_{0}} \vec{a}_{l}(h) T^{l}
$$

with $\left|\vec{a}_{l}(h)\right| \leqslant C_{0}$ satisfies the above stability condition.

(2) The spectral derivative approximation $\nabla^{h, \infty}$ satisfies the stability condition when acting on periodic functions.

We postpone the simple proof of this proposition until the end of this section. Our second requirement on $\nabla_{\alpha}^{h}$ is accuracy. 
Definition. We say that $\nabla_{\alpha}^{h}$ is $r$ th order accurate provided that, for any sufficiently smooth function $w$ with compact support,

$$
\left|\nabla_{\alpha}^{h} w_{j}-\nabla_{\alpha} w(j h)\right|_{0, h} \leqslant C h^{r},
$$

where $C$ depends upon sufficiently many derivatives of $w$.

The antisymmetric difference operators defined in (1.14) are $2 p$-order accurate for each positive integer $p$. Also the spectral derivative approximation $\nabla_{\alpha}^{h, \infty}$ is "infinite order" accurate, i.e., for any integer $r$ and smooth periodic function $w$,

$$
\left|\nabla_{\alpha}^{h, \infty} w_{j}-\nabla_{\alpha} w(j h)\right|_{0, h} \leqslant C_{r} h^{r} .
$$

This fact is well known and is proved in complete detail in Theorem 2.3 and Corollary 2.1 of [15].

Finally, we state our two general theorems.

THEOREM 1 (CONVERGENCE OF FINITE ORDER METHODS). Assume that the velocity field $u(z, t)$ is sufficiently smooth on the time interval $0 \leqslant t \leqslant T$ and that the initial vorticity has compact support. Also assume

(i) the cutoff $\psi$ belongs to $\mathrm{FeS}^{-M, p}$ for some $M$, $p$ with $5<M \leqslant \infty$. If $M=\infty$, then $p \geqslant 4$; if $M<\infty$, then $p$ satisfies $p(M-1) /(p+M)>3$.

(ii) The difference operator $\nabla_{\alpha}^{h}$ is rth order accurate and stable in the sense of (2.3), with $r>3$.

(iii) We choose $\delta=h^{q}$, where $q=1-\varepsilon_{0}$ if $M=\infty$; otherwise

$$
q=\frac{M-1-\varepsilon_{0}}{p+M} .
$$

In both cases $\varepsilon_{0}$ should be small enough so that $p q>3$.

Then we have the following estimates for the quantities computed by the vortex algorithm (1.7)-(1.10):

$$
\begin{gathered}
\max _{0 \leqslant t \leqslant T}\left\{\left|\tilde{z}_{i}(t)-z_{i}(t)\right|_{0, h}+\left|\tilde{\omega}_{i}(t)-\omega_{i}(t)\right|_{-1, h}\right\} \leqslant C\left(h^{p q}+h^{r}\right), \\
\max _{0 \leqslant t \leqslant T}\left|\tilde{u}_{i}^{h}(t)-u_{i}(t)\right|_{0, h} \leqslant C\left(h^{p q}+h^{r}\right), \\
\max _{0 \leqslant t \leqslant T}\left|\tilde{u}^{h}(\cdot, t)-u(\cdot, t)\right|_{L^{2}\left(B\left(R_{0}\right)\right)} \leqslant C\left(h^{p q}+h^{r}\right) .
\end{gathered}
$$

Here $R_{0}>0$ is arbitrary and $B\left(R_{0}\right)=\left\{|z|<R_{0}\right\}$. The constant $C$ depends on $T, M, p, \varepsilon_{0}, r, R_{0}$, the diameter of supp $\omega_{0}$, and bounds for a finite number of derivatives of the velocity field.

THEOREM 2 (CONVERGENCE OF INFINITE ORDER METHODS). Assume further that $\psi \in \mathrm{FeS}^{-\infty, \infty}$ and $\nabla_{\alpha}^{h, \infty}$ is the spectral approximation introduced in (1.18). Choose $\delta=h^{q}, q<1$. Then the quantities computed by the vortex algorithm satisfy the error estimates of Theorem 1 with the right-hand side replaced by $C_{r} h^{r}$ for any $r>0$.

Obviously one should choose $r$ and $p$ so that the respective errors balance, and we have done this for the algorithms defined in the Introduction. We have included the case $M<\infty$ since it explains the necessity of choosing $\delta=h^{q}, q<1$, when the vortex core is not very smooth. We shall have more to say about this in Part II, and our results in 2-D will be considerably sharper. The statement of Theorem 1 can be improved in one respect. It is possible to carry through the arguments here with $L^{2}$ 
norms replaced by $L^{\mu}$ norms, $\mu$ arbitrarily large, with only slight changes. By doing so we can allow any choice of $p, q, r$ so that $p q>1, r>1,0<q<1$, provided $M$ is large enough. Thus we could choose for $\psi$ a function in $\mathrm{FeS}^{-\infty, 2}$, such as a Gaussian distribution, and obtain convergence to essentially second order. Details will be given in the 2-D case in Part II.

The proofs of thse theorems are based primarily on consistency and stability estimates for the velocity approximation, which we now state. The Stability Lemma is proved in Section 4, and the Consistency Lemma is treated in detail in Part II.

Main Stability Lemma. Assume the hypothesis of either Theorem 1 or Theorem 2. Provided that

$$
\max _{0 \leqslant t \leqslant T_{*}}\left|\tilde{z}_{i}-z_{i}\right|_{0, h} \leqslant h^{3}
$$

for some $T_{*} \leqslant T$, we have for $0 \leqslant t \leqslant T_{*}$ the estimates

$$
\begin{gathered}
\left|\tilde{u}_{i}^{h}(t)-u_{i}^{h}(t)\right|_{0, h} \leqslant C\left(\left|\tilde{z}_{i}(t)-z_{i}(t)\right|_{0, h}+\left|\tilde{\omega}_{i}(t)-\omega_{i}(t)\right|_{-1, h}\right), \\
\left|\tilde{u}^{h}(\cdot, t)-u^{h}(\cdot, t)\right|_{L^{2}\left(B\left(R_{0}\right)\right)} \\
\leqslant C\left(\left|\tilde{z}_{i}(t)-z_{i}(t)\right|_{0, h}+\left|\tilde{\omega}_{i}(t)-\omega_{i}(t)\right|_{-1, h}\right) .
\end{gathered}
$$

Main Consistency Lemma. (1) Under the hypothesis of Theorem 1 , for $h \leqslant h_{0}$ and any $R_{0}>0$, we have

$$
\max _{\substack{|z| \leqslant R_{0} \\ 0 \leqslant t \leqslant T}}\left|u^{h}(z, t)-u(z, t)\right| \leqslant C h^{p q} .
$$

Here $u^{h}(z, t)$ is defined as in (1.11).

(2) Under the hypothesis of Theorem 2, the right-hand side of (2.11) can be replaced by $C_{r} h^{r}$ for any $r>0$.

The dependence of the constants in both statements is as described in Theorem 1; in (2.9) and (2.10), $C$ is independent of $T_{*}$. Assuming these two lemmas, it is a simple matter to complete the proof. We discuss only the case $M=\infty, p<\infty$, since the same argument with only minor changes applies to the other cases. All norms in $H_{h}^{0}$ are restricted to the set $\Lambda_{0}^{h}$, where the computed quantities are defined. Nevertheless, it is important that $\left|\tilde{\omega}_{i}-\omega_{i}\right|_{-1, h}$ is thought of as the norm of a function on all of $\Lambda^{h}$ of bounded support.

To derive (2.5) we introduce

$$
e_{i}=\tilde{z}_{i}-z_{i}, \quad w_{i}=\tilde{\omega}_{i}-\omega_{i} .
$$

In analogy with [10], we will obtain a differential inequality for $\left|e_{i}\right|_{0, h}+\left|w_{i}\right|_{-1, h}$. From the ordinary differential equations (1.7), (1.1) we have

$$
\dot{e}_{i}(t)=\tilde{u}_{i}^{h}(t)-u\left(z_{i}, t\right)=\left[\tilde{u}_{i}^{h}(t)-u_{i}^{h}(t)\right]+\left[u^{h}\left(z_{i}, t\right)-u\left(z_{i}, t\right)\right],
$$

recalling (1.11), (1.12). According to the consistency estimate (2.11), the second term on the right is $O\left(h^{p q}\right)=O\left(\delta^{p}\right)$, uniformly in $i$. Since $h i$ is restricted to a fixed bounded set,

$$
\left|u^{h}\left(z_{i}, t\right)-u\left(z_{i}, t\right)\right|_{0, h} \leqslant C \delta^{p}
$$


Under the assumption (2.8) we can apply the stability estimate (2.9) to the first term and obtain

$$
\left|\dot{e}_{i}(t)\right|_{0, h} \leqslant C\left(\delta^{p}+\left|e_{i}(t)\right|_{0, h}+\left|w_{i}(t)\right|_{-1, h}\right)
$$

for $0 \leqslant t \leqslant T_{*}$.

Similarly we have from (1.8) and (1.5)

$$
\begin{aligned}
\dot{w}_{1}(t) & =\nabla_{\alpha}^{h} \tilde{u}_{i}^{h} \cdot \omega_{0}(i h)-\nabla_{\alpha} u\left(z_{i}, t\right) \cdot \omega_{0}(i h) \\
& =\nabla_{\alpha}^{h}\left(\tilde{u}_{i}^{h}-u\left(z_{i}, t\right)\right) \cdot \omega_{0}(i h)+\left(\nabla_{\alpha}^{h}-\nabla_{\alpha}\right) u\left(z_{i}, t\right) \cdot \omega_{0}(i h), \\
& \dot{w}_{i}(t)=\nabla_{\alpha}^{h} \dot{e}_{i} \cdot \omega_{0}(i h)+\left(\nabla_{\alpha}^{h}-\nabla_{\alpha}\right) u\left(z_{i}, t\right) \cdot \omega_{0}(i h) .
\end{aligned}
$$

It is easy to see that multiplication by a $C^{1}$ function is a bounded operator on $H_{h}^{-1}$. We use this fact and the stability condition (2.3) for $\nabla_{\alpha}^{h}$ to estimate the first term:

$$
\left|\nabla_{\alpha}^{h} \dot{e}_{i} \cdot \omega_{0}(i h)\right|_{-1, h} \leqslant C\left|\nabla_{\alpha}^{h} \dot{e}_{i}\right|_{-1, h} \leqslant C^{\prime}\left|\dot{e}_{i}\right|_{0, h} .
$$

(Here we extend $\dot{e}_{i}$ to $\Lambda^{h}$ by defining it to be 0 outside $\Lambda_{0}^{h}$. The extension does not affect the product, since $\omega_{0}(i h)$ has support in $\Lambda_{0}^{h}$.) Because $u$ and $\omega_{0}$ are smooth and $\omega_{0}$ has bounded support, the accuracy condition on $\nabla_{\alpha}^{h}$ implies

$$
\left|\left(\nabla_{\alpha}^{h}-\nabla_{\alpha}\right) u\left(z_{i}, t\right) \cdot \omega_{0}(i h)\right|_{0, h} \leqslant C h^{r} .
$$

Combining this with (2.12)-(2.14) we have finally

$$
\left|\dot{e}_{i}\right|_{0, h}+\left|\dot{w}_{i}\right|_{-1, h} \leqslant C_{0}\left(\delta^{p}+h^{r}+\left|e_{i}\right|_{0, h}+\left|w_{i}\right|_{-1, h}\right)
$$

for $0 \leqslant t \leqslant T_{*}$, where $T_{*}$ is any time such that (2.8) holds. We also have the initial conditions $e_{i}(0)=0, w_{i}(0)=0$. It follows that

$$
\left|e_{i}(t)\right|_{0, h}+\left|w_{i}(t)\right|_{-1, h} \leqslant y(t), \quad 0 \leqslant t \leqslant T_{*},
$$

where $y(t)$ is the solution of

$$
y^{\prime}=C_{0}\left(\delta^{p}+h^{r}+y\right), \quad y(0)=0 .
$$

(E.g., see [12, Section I.6, Lemma 6.1 and Theorem 6.1]; the arguments given there for vector-valued functions apply equally well to functions with values in a Banach space.) Therefore

$$
\left|e_{i}(t)\right|_{0, h}+\left|w_{i}(t)\right|_{-1, h} \leqslant C_{1}\left(\delta^{p}+h^{r}\right)
$$

as long as (2.8) holds. Here $C_{1}$ depends on $C_{0}$ and $T$, but not $T_{*}$.

We can now remove the restriction (2.8). Since $p q, r>3$,

$$
C_{1}\left(\delta^{p}+h^{r}\right) \leqslant \frac{1}{2} h^{3}, \quad h \leqslant h_{0},
$$

for some $h_{0}$. Thus, assuming $h \leqslant h_{0}$, the left-hand side of (2.15) is bounded by $\frac{1}{2} h^{3}$ until the first time it reaches $h^{3}$. But then it can never reach $h^{3}$, so that $(2.8)$ holds with $T_{*}=T$. Therefore $(2.15)$ is true for $0 \leqslant t \leqslant T$.

The first estimate (2.5) of Theorem 1 is now verified. The remaining two estimates follow directly from (2.5): For (2.6), we write

$$
\tilde{u}_{i}^{h}(t)-u_{i}(t)=\left[\tilde{u}_{i}^{h}(t)-u_{i}^{h}(t)\right]+\left[u^{h}\left(z_{i}(t), t\right)-u\left(z_{i}(t), t\right)\right]
$$

and apply the stability estimate (2.9) to the first term and the consistency estimate (2.11) to the second. Similarly

$$
\tilde{u}^{h}(z, t)-u(z, t)=\left[\tilde{u}^{h}(z, t)-u^{h}(z, t)\right]+\left[u^{h}(z, t)-u(z, t)\right] .
$$


Using the continuous stability estimate (2.10) for the first term and the consistency for the second, we obtain (2.7).

Finally, to complete this section, we give the

Proof of Proposition 2.1. To prove (1), we observe that the $i$ th component $D_{\alpha_{i}}^{h}$ of $\nabla_{\alpha}^{h}$ can be decomposed as

$$
D_{\alpha_{i}}^{h}=\sum_{|l| \leqslant l_{1}} b_{i, l}(h) D_{i}^{-} T^{l}
$$

with $\left|b_{i, l}(h)\right| \leqslant C$. We compute

$$
\begin{aligned}
&\left|\left(D_{\alpha_{i}}^{h} w_{j}, g_{j}\right)_{h}\right| \leqslant C \sum_{|\eta| l_{1}+1}\left|\left(T^{l} w, D_{i}^{+} g\right)_{h}\right| \\
& \leqslant C \sum_{\mid \eta \leqslant l_{1}+1}\left|T^{l} w\right|_{0, h}\left|D_{i}^{+} g\right|_{0, h} \leqslant C|w|_{0, h}|g|_{1, h} .
\end{aligned}
$$

Now using the dual pairing of $H_{h}^{-1}$ and $H_{h}^{1}$ and the definition (1.16), we obtain the required estimate in (1) of the proposition. Similarly, (2) follows easily once we verify that for all periodic scalar grid functions,

$$
\left|\left(D_{\alpha_{i}}^{h} w_{j}, g_{j}\right)_{h}\right| \leqslant C\left|w_{j}\right|_{0, h}\left|g_{j}\right|_{1, h},
$$

where $D_{\alpha_{i}}^{h}$ is the spectral derivative approximation to $\partial / \partial \alpha_{i}, i=1,2,3$. (If necessary, we can rescale $\alpha$ so that the support of the initial vorticity $\omega_{0}(\alpha)$ lies inside the unit cube $0<\alpha_{i}<1, i=1,2,3$.) To prove this estimate, without loss of generality, we assume $(2 N+1) h=1$ and use the discrete Fourier transform, computed by

$$
\hat{a}_{r}=\left(a_{j}, e^{2 \pi i r \alpha_{j}}\right)_{h}
$$

where $r=\left(r_{1}, r_{2}, r_{3}\right)$ and $\left|r_{i}\right| \leqslant N$. Then

$$
\left(\widehat{D_{\alpha_{i}}^{h} w_{j}}\right)_{r}=2 \pi i r_{i} \hat{w}_{r}
$$

and, by the discrete Plancherel formula (see [15]),

$$
\begin{aligned}
\left|\left(D_{\alpha_{i}}^{h} w_{j}, g_{j}\right)_{h}\right| & =\left|\sum_{|r|_{1 \infty} \leqslant N} 2 \pi i r_{i} \hat{w}_{r} \bar{g}_{r}\right| \leqslant \sum_{|r|_{1 \infty} \leqslant N}\left|\hat{w}_{r}\right|\left|\frac{\pi \sin \pi h r_{i}}{h}\right|\left|\hat{g}_{r}\right| \\
& \leqslant C\left(\sum\left|\hat{w}_{r}\right|^{2}\right)^{1 / 2}\left(\sum \frac{4 \sin ^{2} \pi h r_{i}}{h^{2}}\left|\hat{g}_{r}\right|^{2}\right)^{1 / 2} \\
& \leqslant C\left|w_{j}\right|_{0, h}\left|D_{i}^{+} g_{j}\right|_{0, h} \leqslant C\left|w_{j}\right|_{0, h}\left|g_{j}\right|_{1, h} .
\end{aligned}
$$

This is the required estimate, and (2) in Proposition 2.1 now follows easily.

3. Continuous and Discrete Integral Operators. The proof of stability will require estimates of integral operators on $L^{2}$-spaces whose kernels are closely related to $K_{\delta}$ of (1.6) and its derivatives. Both discrete and continuous norms occur, since we approximate continuous quantities by representative values. Two versions of the simplest such estimate are given in the following 
LEMMA 3.1. (a) Suppose $\mathcal{K}\left(z, z^{\prime}\right)$ is a $3 \times 3$ matrix depending continuously on $\left(z, z^{\prime}\right) \in S_{1} \times S_{2}$, where $S_{1}$ and $S_{2}$ are bounded subsets of $\mathbf{R}^{3}$. We define the operator $\mathcal{K}: H^{0}\left(S_{2} ; \mathbf{R}^{3}\right) \rightarrow H^{0}\left(S_{1} ; \mathbf{R}^{3}\right)$ by

$$
(\mathscr{K} f)(z)=\int_{S_{2}} \mathscr{K}\left(z, z^{\prime}\right) f\left(z^{\prime}\right) d z^{\prime}, \quad z \in S_{1} .
$$

Then we have

$$
|\mathcal{K} f|_{H^{0}\left(S_{1}\right)} \leqslant\|\mathscr{K}\||f|_{H^{0}\left(S_{2}\right)},
$$

where $\|\mathcal{K}\|$ is the smallest number satisfying

$$
\int_{S_{2}}\left|\mathcal{K}\left(z, y^{\prime}\right)\right| d y^{\prime} \leqslant\|\mathcal{K}\|, \quad \int_{S_{1}}\left|\mathcal{K}\left(y, z^{\prime}\right)\right| d y \leqslant\|\mathscr{K}\|
$$

for all $z \in S_{1}, z^{\prime} \in S_{2}$.

(b) With $S_{1}, S_{2}$ as above, suppose $\mathcal{K}_{i j}$ is a $3 \times 3$ matrix defined for multi-indices with ih $\in S_{1}, j h \in S_{2}$. With $\mathscr{K}: H_{h}^{0}\left(S_{2} ; \mathbf{R}^{3}\right) \rightarrow H_{h}^{0}\left(S_{1} ; \mathbf{R}^{3}\right)$ defined by

$$
(\mathscr{K} f)_{i}=\sum_{j h \in S_{2}} \mathscr{K}_{i j} f_{j} h^{3},
$$

we have

$$
|\mathscr{K} f|_{H^{0}\left(S_{1}\right)} \leqslant\|\mathscr{K}\||f|_{H^{0}\left(S_{2}\right)},
$$

where $\|\mathcal{K}\|$ is the smallest number so that

$$
\sum_{l h \in S_{2}}\left|\mathcal{K}_{i l}\right| h^{3} \leqslant\|\mathcal{K}\|, \quad \sum_{l h \in S_{1}}\left|\mathcal{K}_{l j}\right| h^{3} \leqslant\|\mathcal{K}\|
$$

for all $i, j$, with ih $\in S_{1}, j h \in S_{2}$. (Here $H_{h}^{0}(S)$ means $H_{h}^{0}\left(\Lambda^{h} \cap S\right)$.)

The familiar proof of this fact is reproduced in Section 5. A more general version may be found, e.g., in [9]. We use this lemma in conjunction with the following discrete- $L^{1}$ estimate for $D^{\beta} K_{\delta}, \beta$ being a multi-index.

Lemma 3.2. With time $t$ fixed and $z_{j}=z_{j}(t)$, we have

$$
\sum_{|h|<R} \max _{\left|y_{j}\right| \leqslant C_{0} \delta}\left|D^{\beta} K_{\delta}\left(z-z_{j}+y_{j}\right)\right| h^{3} \leqslant \begin{cases}C, & \beta=0, \\ C|\log \delta|, & |\beta|=1, \\ C \delta^{-1}, & |\beta|=2,\end{cases}
$$

for all $z$ with $|z|<R$, provided $h$ (and thus $\delta$ ) is small enough. Here $C$ depends only on $R, C_{0}$, and bounds for the flow.

In practice we take $R$ large enough to contain the support of $\omega$. This statement is the analogue in three dimensions of Lemma 5 of [10]. It and subsequent lemmas are proved in Section 5. Note that the order of $\delta$ in the estimate (3.5) is the same as in two dimensions; the reason is that the increase in the order of singularity of $t h$ is balanced by an additional radial factor in the volume element.

In applying Lemma 3.2 we use a partition of the fluid domain carried forward in time from the grid imposed initially. With $j=\left(j_{1}, j_{2}, j_{3}\right)$ a multi-integer, let $Q_{j}$ be the cube

$$
Q_{j}=\left\{\alpha \in \mathbf{R}^{3}:-h / 2<\alpha_{i}-j_{i} h \leqslant h / 2, i=1,2,3\right\} .
$$


Then the collection $\left\{Q_{j}: j \in \Lambda\right\}$ partitions $\mathbf{R}^{3}$, and each particle trajectory begins at the center of one cube. At later time $t$, the particles beginning in $Q_{j}$ have evolved to a cell

$$
B_{j}=\Phi^{t}\left(Q_{j}\right) .
$$

Since the flow $\Phi^{t}$ is a diffeomorphism, these cells have diameter uniformly of order $h$, and $\left\{B_{j}: j \in \Lambda\right\}$ again partitions $\mathbf{R}^{3}$. (We are ultimately interested only in a neighborhood of supp $\omega$, but it is convenient for the arguments that follow to extend the partition to all space.) The mapping $\Phi^{t}$ is volume-preserving, since the flow is incompressible, and thus each $B_{j}$ has volume $h^{3}$. Further discussion of properties of the flow and the cells $B_{j}$ is given in [10, Section 2].

We use Lemmas 3.1 and 3.2 together in the applications. Suppose, for example, that a kernel $\mathscr{K}\left(z, z^{\prime}\right)$ defined for $|z|,\left|z^{\prime}\right|<R$ satisfies

$$
\left|\mathfrak{K}\left(z, z^{\prime}\right)\right| \leqslant C M_{i j}^{(\prime)} \quad \text { for } z \in B_{i}, z^{\prime} \in B_{j}
$$

where

$$
M_{i j}^{(l)}=\max _{\substack{\left|y_{i},\right|<C_{0} \delta \\|\beta|=l}}\left|D^{\beta} K_{\delta}\left(z_{i}-z_{j}+y_{i j}\right)\right| .
$$

We can apply Lemma 3.1(a), estimating the integrals of (3.2) uniformly over $B_{j}$ or $B_{i}$, and then use Lemma 3.2 to conclude that $\|\mathscr{K}\|$, with $S_{1}=S_{2}=\{|z|<R\}$, is bounded by the right-hand side of (3.5). The roles of $i$ and $j$ are reversed in the second application of (3.5) to (3.2).

The following $L^{1}$ estimate is closely related to Lemma 3.2.

Lemma 3.3. There is a universal constant $C$ so that

$$
\int_{|z|<R}\left|K_{\delta}(z)\right| d z \leqslant C R .
$$

Because we measure the error in the vorticity in $H^{-1}$ norms, we need a version of Lemma 3.1 for operators from $H^{-1}$ to $H^{0}$, and from $H_{h}^{-1}$ to $H_{h}^{0}$. This is the purpose of the next lemma.

Lemma 3.4. (a) With notation as in Lemma 3.1(a), suppose $S_{2}=\left\{z^{\prime}:\left|z^{\prime}\right|<R+1\right\}$. For $f \in H^{-1}\left(\mathbf{R}^{3}\right)$ with supp $f \subseteq\left\{z^{\prime}:\left|z^{\prime}\right|<R\right\}$ we have

$$
|\mathscr{K} f|_{H^{0}\left(S_{1}\right)} \leqslant C\left(\|\mathcal{K}\|+\left\|D_{z^{\prime}} \mathscr{K}\right\|\right)|f|_{H^{-1}}
$$

with operator norms defined as in (3.2).

(b) With notation as in Lemma 3.1(b), suppose $S_{2}$ is as above. For $f \in H_{h}^{-1}$ with supp $f \subseteq\left\{z^{\prime}:\left|z^{\prime}\right|<R\right\}$, we have

$$
|\mathfrak{K} f|_{H_{h}^{0}\left(S_{1}\right)} \leqslant C\left(\|\mathcal{K}\|+\left\|D_{j}^{h} \mathscr{K}\right\|\right)|f|_{H_{h}^{-1}}
$$

with norms as in (3.4); $D_{j}^{h}$ is the (forward) difference quotient with respect to $j$.

We will also use a form of this lemma for operators from $H_{h}^{-1}$ to $H^{0}\left(S_{1}\right)$, i.e., from discrete to continuous spaces. A statement similar to the above holds with obvious modifications. 
The next two lemmas are concerned with properties of the discrete spaces. The first provides estimates of Sobolev type in these norms.

LEMMA 3.5. (a) For $f \in H_{h}^{0}$ we have

$$
\sup _{j}\left|f_{j}\right| \leqslant h^{-3 / 2}|f|_{0, h}
$$

(b) For $f \in H_{h}^{0}$ and $g \in H_{h}^{-1}$ we have

$$
|f g|_{-1, h} \leqslant C h^{-3 / 2}|f|_{0, h}|g|_{-1, h} \text {. }
$$

Given a function $\left\{f_{j}\right\} \in H_{h}^{-1}$, we can associate, for fixed time $t$, a function $f$ on $\mathbf{R}^{3}$ defined by

$$
f(z)=f_{j}, \quad z \in B_{j}(t) .
$$

We will need the following boundedness property:

LEMMA 3.6. With $\left\{f_{j}\right\}$ and $f$ as above,

$$
|f|_{H^{-1}\left(\mathbf{R}^{3}\right)} \leqslant C\left|f_{j}\right|_{H_{h}^{-1}}
$$

where $C$ depends only on bounds for the flow.

Finally we will use two estimates for $K_{\delta}$ as a convolution kernel. They are based on the observation that the kernel $K$ of the Biot-Savart Law (1.2) is closely related to the Green's function for the Laplacian. Denoting convolution by *, we can express (1.2), (1.3) as

$$
K * \omega=-\nabla \times\left(\Delta^{-1} \omega\right) .
$$

The following estimates are derived in Section 5 from this representation using the Fourier transform.

LEMMA 3.7. For $|\beta|=1$, we have for all $f \in H^{0}$,

$$
\left|\left(D^{\beta} K_{\delta}\right) * f\right|_{0} \leqslant C|f|_{0} .
$$

Lemma 3.8. Suppose $f \in H^{-1}\left(\mathbf{R}^{3}\right)$ and $\operatorname{supp} f \subseteq\{|z|<R\}$, with $R$ fixed. Then

$$
\left|K_{\delta} * f\right|_{H^{0}(|z|<R)} \leqslant C|f|_{H^{-1}\left(\mathbf{R}^{3}\right)}
$$

where $C$ depends only on $R$.

4. The Main Stability Lemma. We are now ready to verify the stability of the discrete approximation to the velocity, as expressed in (2.9) and (2.10). Thus we estimate first the difference between

$$
\tilde{u}_{i}^{h}=\sum_{j} K_{\delta}\left(\tilde{z}_{i}-\tilde{z}_{j}\right) \tilde{\omega}_{j} h^{3}, \quad u_{i}^{h}=\sum_{j} K_{\delta}\left(z_{i}-z_{j}\right) \omega_{j} h^{3}
$$

in terms of $e_{j}=\tilde{z}_{j}-z_{j}, w_{j}=\tilde{\omega}_{j}-\omega_{j}$ under the assumption that

$$
\left|e_{j}\right|_{0, h} \leqslant h^{3} \text {. }
$$

We do not indicate the dependence on the time $t$ since it is fixed. Also we will assume implicitly throughout this argument that $i$ and $j$ are restricted to values so that $i h$ and $j h$ are within the fixed set $\Lambda_{0}^{h}$ for which $\tilde{z}_{j}$ and $\tilde{\omega}_{j}$ are computed. 
In applying Lemma 3.2 we will need a crude uniform bound for $e_{j}$. It follows from (4.1) and Lemma 3.5(a) that $\left|e_{j}\right| \leqslant h^{3 / 2}$. Assuming $h \leqslant 1$, we have $h \leqslant \delta$ in all the cases of Section 2 and thus

$$
\max \left|e_{j}\right| \leqslant \delta
$$

We can write $\tilde{u}_{i}^{h}-u_{i}^{h}$ symbolically as

$$
\begin{aligned}
\tilde{u}_{i}^{h}-u_{i}^{h} & =\tilde{K}_{\delta} \tilde{\omega}-K_{\delta} \omega=\left(\tilde{K}_{\delta}-K_{\delta}\right) \omega+\tilde{K}_{\delta}(\tilde{\omega}-\omega) \\
& =\left(\tilde{K}_{\delta}-K_{\delta}\right) \omega+K_{\delta} w+\left(\tilde{K}_{\delta}-K_{\delta}\right) w \equiv v_{i}^{(1)}+v_{i}^{(2)}+v_{i}^{(3)},
\end{aligned}
$$

where $\tilde{K}_{\delta}$ is evaluated at $\left(\tilde{z}_{i}-\tilde{z}_{j}\right)$, etc. We begin with $v_{i}^{(1)}$, expanding it further to $v_{i}^{(11)}+v_{i}^{(12)}$, where

$$
\begin{aligned}
& v_{i}^{(11)}=\sum_{j}\left[K_{\delta}\left(z_{i}-\tilde{z}_{j}\right)-K_{\delta}\left(z_{i}-z_{j}\right)\right] \omega_{j} h^{3}, \\
& v_{i}^{(12)}=\sum_{j}\left[K_{\delta}\left(\tilde{z}_{i}-\tilde{z}_{j}\right)-K_{\delta}\left(z_{i}-\tilde{z}_{j}\right)\right] \omega_{j} h^{3} .
\end{aligned}
$$

Applying the Mean Value Theorem in (4.3), we have

$$
v_{i}^{(11)}=\sum_{j}\left[\sum_{|\beta|=1} D^{\beta} K_{\delta}\left(z_{i}-z_{j}+y_{i j}\right) e_{j}^{\beta}\right] \omega_{j} h^{3} .
$$

More precisely, we apply the Mean Value Theorem to each component along the line segment from $\left(z_{i}-z_{j}\right)$ to $\left(\tilde{z}_{i}-\tilde{z}_{j}\right)$; we ignore the fact that $y_{i j}$ may depend on the component, since we can treat each separately. Similarly, we ignore the sum over $\beta$ and write

$$
v_{i}^{(11)}=\sum_{j} D K_{\delta}\left(z_{i}-z_{j}+y_{i j}\right) e_{j} \omega_{j} h^{3}
$$

According to (4.2), we can assume

$$
\left|y_{i j}\right| \leqslant \delta
$$

for each $i, j$.

In order to have more flexibility in estimating $v_{i}^{(11)}$, we regard $v_{i}^{(11)}$ and $e_{j} \omega_{j}$ as step functions on $S=\cup\left\{B_{j}:|j h| \leqslant R_{0}\right\}$ and (4.5) as an integral operator on such functions, with $B_{j}$ as in Section 3. Let $v^{(11)}(z)=v_{i}^{(11)}$ for $z \in B_{i}$, and $f\left(z^{\prime}\right)=e_{j} \omega_{j}$ for $z^{\prime} \in B_{j}$. Also let

$$
\mathscr{K}\left(z, z^{\prime}\right)=D K_{\delta}\left(z_{i}-z_{j}+y_{i j}\right) \quad z \in B_{i}, z^{\prime} \in B_{j} .
$$

Then (4.5) is equivalent to

$$
v^{(11)}(z)=\int_{S} \mathcal{K}\left(z, z^{\prime}\right) f\left(z^{\prime}\right) d z^{\prime}, \quad z \in S .
$$

Moreover, since the flow is volume-preserving,

$$
\left|v^{(11)}\right|_{H^{0}(S)}=\left|v_{i}^{(11)}\right|_{0, h}, \quad|f|_{H^{0}(S)}=\left|e_{j} \omega_{j}\right|_{0, h} .
$$

Thus an estimate for (4.7) will apply directly to (4.5).

It is natural to rewrite $\mathcal{K}$ as

$$
\begin{gathered}
\mathscr{K}\left(z, z^{\prime}\right)=\mathscr{K}_{1}\left(z, z^{\prime}\right)+\mathscr{K}_{2}\left(z, z^{\prime}\right), \quad \mathscr{K}_{1}\left(z, z^{\prime}\right)=D K_{\delta}\left(z-z^{\prime}\right), \\
\mathscr{K}_{2}\left(z, z^{\prime}\right)=D K_{\delta}\left(z_{i}-z_{j}+y_{i j}\right)-D K_{\delta}\left(z-z^{\prime}\right) .
\end{gathered}
$$


The $\mathscr{K}_{1}$-term in (4.7) is just $\left(D K_{\delta} * f\right)(z)$, and we have from Lemma $3.6\left|\mathscr{K}_{1} f\right|_{0} \leqslant$ $C|f|_{0}$, or, since $\omega_{j}$ is uniformly bounded, $\left|\mathscr{K}_{1} f\right|_{0} \leqslant C\left|e_{j}\right|_{0, h}$. Using the Mean Value Theorem again, we can estimate

$$
\left|\mathcal{K}_{2}\left(z, z^{\prime}\right)\right| \leqslant M_{i j}^{(2)} \delta, \quad z \in B_{i}, z^{\prime} \in B_{j},
$$

with $M$ as in (3.6). As remarked earlier, it follows from Lemmas 3.1(a) and 3.2 that

$$
\left|\mathcal{K}_{2} f\right|_{0} \leqslant C \delta \cdot \delta^{-1}|f|_{0} \leqslant C^{\prime}\left|e_{j}\right|_{0, h} .
$$

We next apply the Mean Value Theorem to write (4.4) as

$$
v_{i}^{(12)}=\sum_{j} e_{i} D K_{\delta}\left(z_{i}-z_{j}+y_{i j}\right) \omega_{j} h^{3}
$$

with $y_{i j}$ again satisfying (4.6). Since $e_{i}$ factors out of the sum, we will have $\left|v_{i}^{(12)}\right|_{0, h} \leqslant C\left|e_{i}\right|_{0, h}$ provided we establish

$$
\left|\sum_{j} D K_{\delta}\left(z_{i}-z_{j}+y_{i j}\right) \omega_{j} h^{3}\right| \leqslant C
$$

for each $i$. With $i$ fixed, let $g\left(z^{\prime}\right)=\omega_{j}$ and $\mathscr{K}\left(z^{\prime}\right)=D K_{\delta}\left(z_{i}-z_{j}+y_{i j}\right)$ for $z^{\prime} \in B_{j}$. Then the sum above is

$$
\int_{S} \mathscr{K}\left(z^{\prime}\right) g\left(z^{\prime}\right) d z^{\prime}
$$

Now

$$
\left|D K_{\delta}\left(z_{i}-z_{j}+y_{i j}\right)-D K_{\delta}\left(z_{i}-z^{\prime}\right)\right| \leqslant M_{i j}^{(2)} \delta
$$

for $z^{\prime} \in B_{j}$. Thus if we replace $\mathscr{K}\left(z^{\prime}\right)$ by $D K_{\delta}\left(z_{i}-z^{\prime}\right)$ in the integral we commit an error which, according to Lemma 3.2 , is bounded by

$$
C \boldsymbol{\delta} \cdot \delta^{-1} \cdot \max \left|\omega_{j}\right| \leqslant C^{\prime}
$$

Furthermore, in the same way we can replace $g\left(z^{\prime}\right)$ by $\omega\left(z^{\prime}\right)$ with an error bounded by

$$
C h|\log \delta| \max |D \omega| \leqslant C^{\prime} .
$$

The integral is now replaced by

$$
\int_{S} D K_{\delta}\left(z_{i}-z^{\prime}\right) \omega\left(z^{\prime}\right) d z^{\prime}=\int_{S} K_{\delta}\left(z_{i}-z^{\prime}\right) D \omega\left(z^{\prime}\right) d z^{\prime} .
$$

There are no boundary terms in the integration by parts, since $\omega$ is supported in $S$. We estimate this by

$$
\max |D \omega| \cdot \int_{S}\left|K_{\delta}\left(z_{i}-z^{\prime}\right)\right| d z^{\prime}
$$

which, in view of Lemma 3.3, is bounded by a constant. We have now completed the proof that $\left|v_{i}^{(1)}\right|_{0, h} \leqslant C\left|e_{j}\right|_{0, h}$.

We next consider

$$
v_{i}^{(2)}=\sum_{j} K_{\delta}\left(z_{i}-z_{j}\right) w_{j} h^{3} .
$$


The norm $\left|v_{i}^{(2)}\right|_{0, h}$ is the same as $\left|v^{(2)}\right|_{0}$, where $v^{(2)}(z)=v_{i}^{(2)}, z \in B_{i}$. If we also set $w\left(z^{\prime}\right)=w_{j}$ for $z^{\prime} \in B_{j}$ and $\mathcal{K}\left(z, z^{\prime}\right)=K_{\delta}\left(z_{i}-z_{j}\right)$ for $z \in B_{i}, z^{\prime} \in B_{j}$, we can write

$$
v^{(2)}(z)=\int \mathscr{K}\left(z, z^{\prime}\right) w\left(z^{\prime}\right) d z^{\prime} .
$$

In order to estimate this, we expand $\mathscr{K}$ as

$$
\begin{aligned}
\mathscr{K}\left(z, z^{\prime}\right)= & K_{\delta}\left(z-z^{\prime}\right)+\left[K_{\delta}\left(z_{i}-z_{j}\right)-K_{\delta}\left(z_{i}-z^{\prime}\right)\right] \\
& +\left[K_{\delta}\left(z_{i}-z^{\prime}\right)-K_{\delta}\left(z-z^{\prime}\right)\right] \\
\equiv & \mathscr{K}_{1}+\mathscr{K}_{2}+\mathscr{K}_{3} .
\end{aligned}
$$

We denote the corresponding terms of $v^{(2)}$ by $v^{(21)}, v^{(22)}, v^{(23)}$. The first is just $K_{\delta} * w$, so that by Lemma 3.8, $\left|v^{(21)}\right|_{0} \leqslant C|w|_{-1}$. Combining this with Lemma 3.6 we have $\left|v^{(21)}\right|_{0} \leqslant C\left|w_{j}\right|_{-1, h}$. The term $v^{(22)}$ has the form

$$
v^{(22)}\left(z_{i}\right)=\sum_{j} \mathscr{K}_{2 i j} w_{j} h^{3},
$$

where

$$
\mathscr{K}_{2 i j}=h^{-3} \int_{B_{j}}\left[K_{\delta}\left(z_{i}-z_{j}\right)-K_{\delta}\left(z_{i}-z^{\prime}\right)\right] d z^{\prime} .
$$

We will think of this as an operator from $H_{h}^{-1}$ to $H_{h}^{0}$ and estimate using Lemma 3.4(b). Since we have to difference in $j$, we refrain from estimating $\mathscr{K}_{2 i j}$ directly. It will be convenient to change the variable of integration; we set $z^{\prime}=\Phi^{t}\left(\alpha^{\prime}\right)$ and, with $i$ fixed, $F\left(\alpha^{\prime}\right)=K_{\delta}\left(z_{i}-\Phi^{t}\left(\alpha^{\prime}\right)\right)$. Then

$$
\mathscr{K}_{2 i j}=h^{-3} \int_{Q_{j}}\left[F\left(\alpha_{j}\right)-F\left(\alpha^{\prime}\right)\right] d \alpha^{\prime}=h^{-3} \int_{Q_{0}}\left[F\left(\alpha_{j}\right)-F\left(\alpha_{j}+\alpha^{\prime}\right)\right] d \alpha^{\prime},
$$

where $Q_{0}$ is the cube centered at the origin. We write the integrand as the integral of its derivative:

$$
F\left(\alpha_{j}\right)-F\left(\alpha_{j}+\alpha^{\prime}\right)=-\int_{0}^{1} \nabla F\left(\alpha_{j}+s \alpha^{\prime}\right) d s \cdot \alpha^{\prime}
$$

This gives us an estimate for the kernel $\mathcal{K}_{2 i j}$ :

$$
\left|F\left(\alpha_{j}\right)-F\left(\alpha_{j}+\alpha^{\prime}\right)\right| \leqslant h \sup _{Q_{j}}|D F| \leqslant C h \sup _{z^{\prime} \in B_{j}}\left|D K_{\delta}\left(z_{i}-z^{\prime}\right)\right| \leqslant C h M_{i j}^{(1)}
$$

by the smoothness of $\Phi^{t}$, and therefore $\left|\mathcal{K}_{2 i j}\right| \leqslant C h M_{i j}^{(2)}$. It follows from Lemma 3.2 that $\left\|\mathcal{K}_{2 i j}\right\| \leqslant C h|\log \delta| \leqslant C$, with $\left\|\mathscr{K}_{2 i j}\right\|$ as in (3.4). However, we must also estimate $\left\|D_{j}^{h} \mathcal{K}_{2 i j}\right\|$. We have

$$
\begin{gathered}
D_{j}^{h} \mathcal{K}_{2 i j}=h^{-3} \int_{Q_{0}} D_{j}^{h}\left[F\left(\alpha_{j}\right)-F\left(\alpha_{j}+\alpha^{\prime}\right)\right] d \alpha^{\prime}, \\
D_{j}^{h}\left[F\left(\alpha_{j}\right)-F\left(\alpha_{j}+\alpha^{\prime}\right)\right]=-\int_{0}^{1} D_{h}\left[\nabla F\left(\alpha_{j}+s \alpha^{\prime}\right)\right] d s \cdot \alpha^{\prime} .
\end{gathered}
$$

Thus

$$
\left|D_{j}^{h}\left[F\left(\alpha_{j}\right)-F\left(\alpha_{j}+\alpha^{\prime}\right)\right]\right| \leqslant h \sup _{Q_{j} \cup Q_{j+1}}\left|D^{2} F\right| \leqslant C h M_{i j}^{(2)}
$$


where $Q_{j+1}$ denotes a cube adjacent to $Q_{j}$, and $\left|D_{j}^{h} \mathcal{K}_{2 i j}\right| \leqslant C h M_{i j}^{(2)}$. Finally, we conclude from Lemma 3.2 that $\left\|D_{j}^{h T K_{2 i j}}\right\| \leqslant C h \cdot \delta^{-1} \leqslant C$ and therefore, from Lemma 3.4(b),

$$
\left|v^{(22)}\right|_{0}=\left|v^{(22)}\left(z_{i}\right)\right|_{0, h} \leqslant C\left|w_{j}\right|_{-1, h}
$$

To estimate

$$
v^{(23)}(z)=\int \mathscr{K}_{3}\left(z, z^{\prime}\right) w\left(z^{\prime}\right) d z^{\prime}
$$

where

$$
\mathscr{K}_{3}\left(z, z^{\prime}\right)=K_{\delta}\left(z_{i}-z^{\prime}\right)-K_{\delta}\left(z-z^{\prime}\right), \quad z \in B_{i},
$$

we will treat $z$ and $z^{\prime}$ as continuous variables. According to Lemma 3.4(a),

$$
\left|v^{(23)}\right|_{0} \leqslant C|w|_{-1}
$$

provided we show that $\left\|\mathcal{K}_{3}\right\|+\left\|D_{z^{\prime}} \mathscr{K}_{3}\right\| \leqslant C$, with notation as in (3.2). For $z \in B_{i}$, $z^{\prime} \in B_{j},\left|\mathscr{K}_{3}\left(z, z^{\prime}\right)\right| \leqslant h M_{i j}^{(1)}$, and, from Lemma 3.2, $\left\|\mathscr{K}_{3}\right\| \leqslant C h|\log \delta| \leqslant C$. Similarly, $\left|D_{z^{\prime}}, K_{3}\left(z, z^{\prime}\right)\right| \leqslant h M_{i j}^{(2)}$ so that $\left\|D_{z^{\prime}}, K_{3}\right\| \leqslant C h \delta^{-1} \leqslant C$. Thus (4.9) is established. Then, from Lemma 3.6, $\left|v^{(23)}\right|_{0} \leqslant C\left|w_{j}\right|_{-1, h}$.

It remains to estimate

$$
v_{i}^{(3)}=\sum_{j}\left[K_{\delta}\left(\tilde{z}_{i}-\tilde{z}_{j}\right)-K_{\delta}\left(z_{i}-z_{j}\right)\right] w_{j} h^{3} .
$$

We will again apply Lemma 3.4(b). Substituting

$$
K_{\delta}\left(\tilde{z}_{i}-\tilde{z}_{j}\right)-K_{\delta}\left(z_{i}-z_{j}\right)=\mathcal{K}_{i j} \cdot\left(e_{i}-e_{j}\right)
$$

with

$$
\mathcal{K}_{i j}=\int_{0}^{1} \nabla K_{\delta}\left(z_{i}-z_{j}+s\left(e_{i}-e_{j}\right)\right) d s
$$

we have

$$
v_{i}^{(3)}=\sum_{j} \mathcal{K}_{i j} e_{i} w_{j} h^{3}-\sum_{j} \mathscr{K}_{i j} e_{j} w_{j} h^{3} \equiv v_{i}^{(31)}-v_{i}^{(32)} .
$$

For the first part, we find

$$
\left|v_{i}^{(3)}\right|_{0, h} \leqslant\left(\max \left|e_{i}\right|\right)\left|\sum_{j} \mathcal{K}_{i j} w_{j} h^{3}\right|_{0, h} \leqslant \delta\left(\left\|\mathcal{K}_{i j}\right\|+\left\|D_{j}^{h} \mathcal{K}_{i j}\right\|\right)\left|w_{j}\right|_{-1, h}
$$

using (4.2) and Lemma 3.4(b). The difference quotient goes through the $s$-integral, and $\left|\mathcal{K}_{i j}\right| \leqslant M_{i j}^{(1)},\left|D_{j}^{h} \mathcal{K}_{i j}\right| \leqslant M_{i j}^{(2)}$, so that, by Lemma 3.2,

$$
\left|v_{i}^{(3)}\right|_{0, h} \leqslant C \delta\left(|\log \delta|+\delta^{-1}\right)\left|w_{j}\right|_{-1, h} \leqslant 2 C\left|w_{j}\right|_{-1, h} .
$$

For $v_{i}^{(32)}$, the same kernel $\mathscr{K}_{i j}$ is applied to the function $e_{j} w_{j}$. We use Lemma 3.5(b) and (4.1):

$$
\left|e_{j} w_{j}\right|_{-1, h} \leqslant C h^{-3 / 2}\left|e_{j}\right|_{0, h}\left|w_{j}\right|_{-1, h} \leqslant C h^{3 / 2}\left|w_{j}\right|_{-1, h} .
$$

Thus

$$
\begin{aligned}
\left|v_{i}^{(32)}\right|_{0, h} & \leqslant\left(\left\|\mathcal{K}_{i j}\right\|+\left\|D_{j}^{h \mathcal{K}} \mathcal{K}_{i j}\right\|\right) \|\left. e_{j} w_{j}\right|_{-1, h} \\
& \leqslant C\left(|\log \delta|+\delta^{-1}\right) h^{3 / 2}\left|w_{j}\right|_{-1, h} \leqslant C\left|w_{j}\right|_{-1, h} .
\end{aligned}
$$

This completes the proof of (2.9). 
The estimate (2.10) is established by a similar argument. There are fewer terms, since there is no error in the point $z$ at which the velocity approximation is evaluated. With

$$
\tilde{u}^{h}(z)=\sum_{j} K_{\delta}\left(z-\tilde{z}_{j}\right) \tilde{\omega}_{j} h^{3}, \quad u^{h}(z)=\sum_{j} K_{\delta}\left(z-z_{j}\right) \omega_{j} h^{3},
$$

we write as before

$$
\tilde{u}^{h}-u^{h}=\left(\tilde{K}_{\delta}-K_{\delta}\right) \omega+K_{\delta} w+\left(\tilde{K}_{\delta}-K_{\delta}\right) w \equiv v^{(1)}+v^{(2)}+v^{(3)}
$$

The term

$$
v^{(1)}(z)=\sum_{j}\left[K_{\delta}\left(z-\tilde{z}_{j}\right)-K_{\delta}\left(z-z_{j}\right)\right] \omega_{j} h^{3}
$$

is treated like the earlier term $v^{(11)}$. We write it in the form (4.7), with $f$ as before and

$$
\begin{gathered}
\mathscr{K}\left(z, z^{\prime}\right)=D K_{\delta}\left(z-z^{\prime}\right)+\mathscr{K}_{2}\left(z, z^{\prime}\right), \\
\mathcal{K}_{2}\left(z, z^{\prime}\right)=D K_{\delta}\left(z-z_{j}+y_{j}\right)-D K_{\delta}\left(z-z^{\prime}\right), \quad z^{\prime} \in B_{j},
\end{gathered}
$$

where $y_{j}$ depends on $z,\left|y_{j}\right| \leqslant \delta$. The first part of $v^{(1)}$ is again $K_{\delta} * f$, and the second part is estimated just as before.

For $v^{(2)}$, we begin with the expression (4.8), where

$$
\begin{gathered}
\mathscr{K}\left(z, z^{\prime}\right)=K_{\delta}\left(z-z^{\prime}\right)+\mathscr{K}_{2}\left(z, z^{\prime}\right), \\
\mathscr{K}_{2}\left(z, z^{\prime}\right)=K_{\delta}\left(z-z_{j}\right)-K_{\delta}\left(z-z^{\prime}\right), \quad z^{\prime} \in B_{j} .
\end{gathered}
$$

The first term in $v^{(2)}$ is the same as earlier; the second has the form

$$
v^{(22)}(z)=\sum_{j} \mathscr{K}_{2 j}(z) w_{j} h^{3}
$$

with

$$
\mathscr{K}_{2 j}(z)=h^{-3} \int_{B_{j}}\left[K_{\delta}\left(z-z_{j}\right)-K_{\delta}\left(z-z^{\prime}\right)\right] d z^{\prime}
$$

We estimate this term by applying a variant of Lemma 3.4(b):

$$
\left|v^{(22)}\right|_{0} \leqslant\left(\left\|\mathcal{K}_{2 j}\right\|+\left\|D_{j}^{h} \mathcal{K}_{2 j}\right\|\right)\left|w_{j}\right|_{-1, h} .
$$

Here the operator norm is as in (3.4), except that the second sum is replaced by integration in $z$. The kernel $\mathscr{K}_{2 j}$ can be estimated in the same way as $\mathscr{K}_{2 i j}$ in the previous case.

The term $v^{(3)}$ takes the form

$$
v^{(3)}(z)=\sum_{j} \mathscr{K}_{j}(z) e_{j} w_{j}, \quad \mathscr{K}_{j}(z)=\int_{0}^{1} \nabla K_{\delta}\left(z-z_{j}-s e_{j}\right) d s .
$$

We estimate as for $v_{i}^{(32)}$ earlier, except that the same modification of Lemma 3.4 as above is used.

\section{Proofs of the Lemmas.}

Proof of Lemma 3.1. Parts (a) and (b) can be regarded as different cases of a general statement in which the measure spaces are arbitrary; e.g., see [9, Section 
0.C]. We include here the proof of (a) for completeness:

$$
\begin{aligned}
\int \mid \int \mathcal{K}\left(z, z^{\prime}\right) & \left.f\left(z^{\prime}\right) d z^{\prime}\right|^{2} d z \\
& \leqslant \int\left\{\int\left|\mathcal{K}\left(z, z^{\prime}\right)\right| d z^{\prime} \cdot \int\left|\mathcal{K}\left(z, z^{\prime}\right)\right|\left|f\left(z^{\prime}\right)\right|^{2} d z^{\prime}\right\} d z \\
& \leqslant\|\mathscr{K}\| \iint\left|\mathcal{K}\left(z, z^{\prime}\right)\right|\left|f\left(z^{\prime}\right)\right|^{2} d z^{\prime} d z \\
& =\|\mathscr{K}\| \int\left\{\int\left|\mathcal{K}\left(z, z^{\prime}\right)\right| d z\right\}\left|f\left(z^{\prime}\right)\right|^{2} d z^{\prime} \\
& \leqslant\|\mathscr{K}\|^{2} \int\left|f\left(z^{\prime}\right)\right|^{2} d z^{\prime} .
\end{aligned}
$$

To prove Lemma 3.2, we need the following pointwise estimates for $D^{\beta} K_{\delta}$ :

LEMMA 5.1. We have

$$
\begin{array}{ll}
\left|D^{\beta} K_{\delta}(z)\right| \leqslant C \delta^{-2-|\beta|}, & \text { all } z, \\
\left|D^{\beta} K_{\delta}(z)\right| \leqslant C|z|^{-2-|\beta|}, & |z| \geqslant \delta .
\end{array}
$$

Proof. For $|z| \geqslant \delta$, (5.2) will imply (5.1). We first prove (5.1) for $|z| \leqslant \delta$. We write

$$
D^{\beta} K_{\delta}(z)=\int K\left(z^{\prime}\right) D^{\beta} \psi_{\delta}\left(z-z^{\prime}\right) d z^{\prime} \equiv I_{1}+I_{2},
$$

where $I_{1}$ is the integral over $\left\{\left|z^{\prime}\right|<2 \delta\right\}$ and $I_{2}$ over $\left\{\left|z^{\prime}\right|>2 \delta\right\}$. For any $\rho>0$, we can easily estimate $K$ in $L^{\prime}\left(\left|z^{\prime}\right|<\rho\right):\left|K\left(z^{\prime}\right)\right|$ is a constant times $\left|z^{\prime}\right|^{-2}$, and in spherical coordinates we have

$$
\int_{\left|z^{\prime}\right|<\rho}\left|K\left(z^{\prime}\right)\right| d z^{\prime}=A \int_{0}^{\rho} r^{-2} \cdot r^{2} d r=A \rho,
$$

where $A$ is a universal constant. Now

$$
D^{\beta} \psi_{\delta}\left(z-z^{\prime}\right)=\delta^{-3-|\beta|}\left(D^{\beta} \psi\right)\left(\left(z-z^{\prime}\right) / \delta\right)
$$

and thus

$$
\left|D^{\beta} \psi_{\delta}\left(z-z^{\prime}\right)\right| \leqslant C \delta^{-3-|\beta|} .
$$

Using these two facts, we have

$$
\left|I_{1}\right| \leqslant C A(2 \delta) \cdot \delta^{-3-|\beta|}=C^{\prime} \delta^{-2-|\beta|} .
$$

On the set $\left\{\left|z^{\prime}\right|>2 \delta\right\}$ we have $\left|z^{\prime}-z\right| \geqslant\left|z^{\prime}\right|-\delta \geqslant \delta$. Since $\psi$ is rapidly decreasing, we have $\left|D^{\beta} \psi(x)\right| \leqslant C|x|^{-2},|x| \geqslant 1$, and therefore, using (5.4),

$$
\left|D^{\beta} \psi_{\delta}\left(z-z^{\prime}\right)\right| \leqslant C \delta^{-3-|\beta|} \delta^{2}\left|z-z^{\prime}\right|^{-2} \leqslant C \delta^{-1-|\beta|}\left(\left|z^{\prime}\right|-\delta\right)^{-2} .
$$

We now estimate $I_{2}$ using this and the relation $K\left(z^{\prime}\right) \sim\left|z^{\prime}\right|^{-2}$ :

$$
\begin{aligned}
\left|I_{2}\right| & \leqslant C \delta^{-1-|\beta|} \int_{2 \delta}^{\infty} r^{-2}(r-\delta)^{-2} r^{2} d r \\
& =C \delta^{-1-|\beta|} \int_{\delta}^{\infty} r^{-2} d r=C \delta^{-2-|\beta|}
\end{aligned}
$$

This completes (5.1). 
Assuming now that $|z| \geqslant \delta$, we prove (5.2) by estimating (5.3) in two parts. Let $\phi_{0}(r)$ be a smooth function so that $\phi_{0}(r)=0$ for $r \leqslant 1 / 4, \phi_{0}(r)=1$ for $r \geqslant 1 / 2$, and $0 \leqslant \phi_{0} \leqslant 1$. With $z$ fixed, let $\phi\left(z^{\prime}\right)=\phi_{0}\left(\left|z^{\prime}\right| /|z|\right)$. We now denote by $I_{1}$ and $I_{2}$ the integrals with $K$ replaced by $\phi K$ and $(1-\phi) K$. In the first term, the singularity at $z^{\prime}=0$ has been removed, and we can write

$$
I_{1}= \pm \int D^{\beta}\left[\phi\left(z^{\prime}\right) K\left(z^{\prime}\right)\right] \cdot \psi_{\delta}\left(z-z^{\prime}\right) d z^{\prime} .
$$

Now $\left|D^{\beta} \phi\right| \leqslant C|z|^{-\beta}$, and $\left|D^{\gamma} K\left(z^{\prime}\right)\right| \leqslant C\left|z^{\prime}\right|^{-2-|\gamma|}$; on the support of $\phi,\left|z^{\prime}\right| \geqslant$ $|z| / 4$, and therefore $\left|D^{\beta}(\phi K)\left(z^{\prime}\right)\right| \leqslant C|z|^{-2-|\beta|}$. Since $\psi_{\delta}$ is bounded in $L^{1}$ independent of $\delta$, we can conclude from (5.5) that $\left|I_{1}\right| \leqslant C|z|^{-2-|\beta|}$.

It remains to show that the same holds for $I_{2}$. It will be enough to establish the estimate

$$
\left|\int_{\left|z^{\prime}\right|<|z| / 2} K\left(z^{\prime}\right) D^{\beta} \psi_{\delta}\left(z-z^{\prime}\right) d z^{\prime}\right| \leqslant C|z|^{-2-|\beta|} .
$$

On this set $\left|z-z^{\prime}\right| \geqslant|z| / 2 \geqslant \delta / 2$. Again, since $\psi$ is rapidly decreasing, we may assume $\left|D^{\beta} \psi(x)\right| \leqslant C_{N}|x|^{-N},|x| \geqslant \frac{1}{2}$, and thus, as in the previous part, $\left|D^{\beta} \psi_{\delta}\left(z-z^{\prime}\right)\right| \leqslant C \delta^{-3-|\beta|} \delta^{N}|z|^{-N}$. Choosing $N=3+|\beta|$ to eliminate $\delta$, we now estimate the integral of $(5.6)$ by

$$
C|z|^{-3-|\beta|} \int_{\left|z^{\prime}\right|<|z| / 2}\left|K\left(z^{\prime}\right)\right| d z^{\prime}=C^{\prime}|z|^{-2-|\beta|} .
$$

Proof of Lemma 3.2. Given the uniform estimate of Lemma 5.1, the proof is virtually the same as that of Lemma 5 in [10]. The diameter of $B_{j}$ is of order $h$, and, since in all cases we have $h \leqslant \delta$, we can assume $\left|z^{\prime}-z_{j}\right| \leqslant C_{1} \delta, z^{\prime} \in B_{j}$, for some $C_{1}$; we might as well assume $C_{1} \geqslant C_{0}$. With $z$ fixed, let

$$
\begin{aligned}
& J_{1}=\left\{j \in \Lambda:|j h|<R,\left|z_{j}-z\right| \leqslant\left(3 C_{1}+1\right) \delta\right\}, \\
& J_{2}=\left\{j \in \Lambda:|j h|<R,\left|z_{j}-z\right|>\left(3 C_{1}+1\right) \delta\right\} .
\end{aligned}
$$

Then

$$
\cup\left\{B_{j}: j \in J_{1}\right\} \subseteq\left\{z^{\prime}:\left|z^{\prime}-z\right| \leqslant\left(4 C_{1}+1\right) \delta\right\} .
$$

Since each $B_{j}$ has volume $h^{3}$, we may use this fact and (5.1) to estimate the part of the sum in (3.5) over $J_{1}$ by

$$
C \delta^{-2-|\beta|}\left\{\left(4 C_{1}+1\right) \delta\right\}^{3}=C^{\prime} \delta^{1-|\beta|} .
$$

This establishes the inequality (3.5) for cells $B_{j}$ with $j \in J_{1}$.

For $j \in J_{2}$, we have $\left|z-z_{j}+y_{j}\right| \geqslant\left(2 C_{1}+1\right) \delta \geqslant \delta$ and, for $z^{\prime} \in B_{j}$,

$$
\left|z-z_{j}+y_{j}\right| \geqslant\left|z-z^{\prime}\right|-2 C_{1} \delta
$$

and also

$$
\left|z-z^{\prime}\right| \geqslant\left|z-z_{j}\right|-\left|z_{j}-z^{\prime}\right| \geqslant\left(1+2 C_{1}\right) \delta .
$$

We regard the sum (3.5) over $J_{2}$ as the integral of a step function with constant value on each $B_{j}$. Using (5.2) and (5.7) we estimate the integrand by

$$
\max _{\left|y_{j}\right| \leqslant C_{0} \delta}\left|D^{\beta} K_{\delta}\left(z-z_{j}+y_{j}\right)\right| \leqslant C\left(\left|z-z^{\prime}\right|-2 C_{1} \delta\right)^{-2-|\beta|} \text {. }
$$


In view of (5.8), the region of integration is contained in $\left\{z^{\prime}:\left(1+2 C_{1}\right) \delta \leqslant\right.$ $\left.\left|z^{\prime}-z\right| \leqslant R_{1}\right\}$ for some $R_{1}$ depending on $R$. Setting $r=\left|z^{\prime}-z\right|$, we can now estimate this part of (3.5) by

$$
C \int_{\left(1+2 C_{1}\right) \delta}^{R_{1}}\left(r-2 C_{1} \delta\right)^{-2-|\beta|} r^{2} d r=C \int_{\delta}^{R_{1}-2 C_{1} \delta} r^{-2-|\beta|}\left(r+2 C_{1} \delta\right)^{2} d r .
$$

But $r+2 C_{1} \delta \leqslant\left(1+2 C_{1}\right) r$ for $r \geqslant \delta$, and the integral is

$$
\leqslant \int_{\delta}^{R_{1}-2 C_{1} \delta} r^{-|\beta|} d r
$$

which leads to the cases of (3.5).

Proof of Lemma 3.3. This follows immediately from Lemma 5.1, applying (5.1) for $|z| \leqslant \delta$ and (5.2) for $|z| \geqslant \delta$.

Proof of Lemma 3.4. The two cases are similar, and we discuss only (a). Let $\phi\left(z^{\prime}\right)$ be a smooth function so that $\phi=1$ for $\left|z^{\prime}\right| \leqslant R, \phi=0$ for $\left|z^{\prime}\right| \geqslant R+1$. In order to estimate $|\mathcal{K} f|_{0}$, we take the inner product with an arbitrary $g \in H^{0}\left(S_{1}\right)$ :

$$
(\mathscr{K} f, g)=\left(f, \mathscr{K}^{*} g\right)=\left(\phi f, \mathscr{K}^{*} g\right)=\left(f, \phi \mathscr{K}^{*} g\right) \text {, }
$$

so that

$$
|(\mathscr{K} f, g)| \leqslant|f|_{-1}\left|\phi K^{*} g\right|_{1}
$$

Here

$$
\left(\mathscr{K}^{*} g\right)\left(z^{\prime}\right)=\int \mathcal{K}\left(z, z^{\prime}\right) g(z) d z
$$

To estimate $\left|\phi \mathcal{K}^{*} g\right|_{1}$, we apply Lemma 3.1(a), with the roles of $z$ and $z^{\prime}$ reversed, to the integral operator

$$
g(z) \mapsto \int D_{z^{\prime}}^{\beta}\left\{\phi\left(z^{\prime}\right) \mathscr{K}\left(z, z^{\prime}\right)\right\} g(z) d z,
$$

where $|\beta|=0$ or 1 . We conclude from Lemma 3.1 that

$$
\left|\phi \mathcal{K}^{*} g\right|_{1} \leqslant C\left(\|\mathcal{K}\|+\left\|D_{z^{\prime}} \mathscr{K}\right\|\right)|g|_{0},
$$

and the assertion follows from this and (5.9).

Proof of Lemma 3.5. Part (a) follows from the crude estimate

$$
\left|f_{j}\right|^{2} h^{3} \leqslant \sum_{i}\left|f_{i}\right|^{2} h^{3}=|f|_{0, h}^{2}
$$

For (b) we first estimate $|f \phi|_{1}$ for $\phi \in H_{h}^{1}$. We have

$$
D^{h}\left(f_{j} \phi_{j}\right)=f_{j}\left(D^{h} \phi_{j}\right)+\phi_{j+1}\left(D^{h} f_{j}\right)
$$

where $\phi_{j+1}$ means $\phi_{j}$ translated in the appropriate coordinate direction. For the first term we use (a):

$$
\begin{aligned}
& \left|f_{j}\left(D^{h} \phi_{j}\right)\right|_{0, h} \leqslant\left(\sup _{j}\left|f_{j}\right|\right) \cdot\left|D^{h} \phi_{j}\right|_{0, h}, \\
& \left|f_{j}\left(D^{h} \phi_{j}\right)\right|_{0, h} \leqslant h^{-3 / 2}\left|f_{j}\right|_{0, h}\left|\phi_{j}\right|_{1, h} .
\end{aligned}
$$

For the second we have

$$
\left|\phi_{j+1}\left(D^{h} f_{j}\right)\right|_{0, h} \leqslant\left(\sup _{j}\left|\phi_{j}\right|\right) \cdot\left|f_{j}\right|_{1, h} \leqslant\left(\sup _{j}\left|\phi_{j}\right|\right) \cdot h^{-1}\left|f_{j}\right|_{0, h} .
$$


To estimate $\phi_{j}$ uniformly, we use a discrete Sobolev inequality [23, p. 181, Chapter II.2, Theorem 2.1] which implies that

$$
\left[\sum_{j}\left|\phi_{j}\right|^{6} h^{3}\right]^{1 / 6} \leqslant C\left|D^{h} \phi\right|_{0, h} .
$$

Thus, as in (a), sup $\left|\phi_{j}\right| \leqslant C h^{-1 / 2}|\phi|_{1, h}$. Combining this with the previous inequalities, we have

$$
\left|D^{h}(f \phi)\right|_{0, h} \leqslant C h^{-3 / 2}|f|_{0, h}|\phi|_{1, h} .
$$

We can treat $|f \phi|_{0, h}$ as in (5.10) and conclude finally that

$$
|f \phi|_{1, h} \leqslant C h^{-3 / 2}|f|_{0, h}|\phi|_{1, h} .
$$

Assertion (b) follows from this by duality: for arbitrary $\phi \in H_{1}^{h}$,

$$
\begin{aligned}
\left|(f g, \phi)_{0, h}\right| & =\left|(g, f \phi)_{0, h}\right| \leqslant|g|_{-1, h}|f \phi|_{1, h} \\
& \leqslant C h^{-3 / 2}|f|_{0, h}|g|_{-1, h}|\phi|_{1, h} .
\end{aligned}
$$

Proof of Lemma 3.6. We have to show that, for any $g \in H^{1}\left(\mathbf{R}^{3}\right)$,

$$
\left|(f, g)_{0}\right| \leqslant C\left|f_{j}\right|_{-1, h}|g|_{1} \text {. }
$$

Given $g$, we define

$$
g_{j}=h^{-3} \int_{B_{j}} g(z) d z
$$

Then

$$
(f, g)_{0}=\int f g d z=\sum_{j} f_{j} \int_{B_{j}} g d z=\sum f_{j} g_{j} h^{3},
$$

so that $\left|(f, g)_{0}\right| \leqslant\left|f_{j}\right|_{-1, h}\left|g_{j}\right|_{1, h}$. Therefore (5.11) will be established if we show

$$
\left|g_{j}\right|_{1, h} \leqslant C|g|_{1} \text {. }
$$

As a first step, we have

$$
\left|g_{j}\right|_{0, h}^{2}=\sum\left|g_{j}\right|^{2} h^{3}=\sum h^{-3}\left|\int_{B_{j}} g\right|^{2} \leqslant \sum h^{-3} \cdot h^{3} \int_{B_{j}}|g|^{2}=|g|_{0}^{2},
$$

using the Schwarz inequality. We next consider the difference quotient

$$
D^{h} g_{j}=h^{-4}\left\{\int_{B_{j+1}} g-\int_{B_{j}} g\right\}
$$

where $j+1$ means $j$ incremented in some direction. For simplicity we assume that the difference is with respect to $z_{1}, z=\left(z_{1}, z_{2}, z_{3}\right)$, and the cube $Q_{j}$ at time 0 was centered at the origin. Then

$$
D^{h} g_{j}=h^{-4} \int_{Q_{j}}\left[G\left(z_{1}+h, z_{2}, z_{3}\right)-G\left(z_{1}, z_{2}, z_{3}\right)\right] d z
$$

with $G=g \circ \Phi^{t}$. Writing $G$ as the integral of $D_{z_{1}} G$, we have

$$
D^{h} g_{j}=h^{-4} \int_{Q_{j}} \int_{z_{1}}^{z_{1}+h} D_{z_{1}} G\left(\rho, z_{2}, z_{3}\right) d \rho d z
$$


Next we estimate, taking absolute values and increasing the $\rho$-interval to $-h / 2 \leqslant \rho$ $\leqslant 3 h / 2$. The $z_{1}$-integral gives a factor of $h$; we combine $d \rho$ with $d z_{2} d z_{3}$ to obtain

$$
\left|D^{h} g_{j}\right| \leqslant C h^{-3} \int_{Q_{j} \cup Q_{j+1}}|D G| d z .
$$

Using the smoothness of the flow, we can express this as

$$
\left|D^{h} g_{j}\right| \leqslant C h^{-3} \int_{B_{j} \cup B_{j+1}}|D g| d z
$$

By an estimate like (5.13), this implies $\left|D^{h} g_{j}\right|_{0, h} \leqslant C|D g|_{0}$, and (5.12) is now verified.

Proof of Lemma 3.7. We have

$$
\left(D^{\beta} K_{\delta}\right) * f=D^{\beta}\left(K * \psi_{\delta}\right) * f=\left(D^{\beta} K\right) *\left(\psi_{\delta} f\right)
$$

so that, using (3.10),

$$
\left[\left(D^{\beta} K_{\delta}\right) * f\right]^{\wedge}(\xi)=(2 \pi i \xi)^{\beta} \hat{\psi}_{\delta}(\xi) \cdot|\xi|^{-2}(2 \pi i \xi) \times \hat{f}(\xi) .
$$

Since $\hat{\psi}_{\delta}(\xi)=\hat{\psi}(\delta \xi)$, we can estimate

$$
\left|\hat{\psi}_{\delta}(\xi)\right| \leqslant|\psi|_{L^{1}}
$$

and therefore, for $|\beta|=1$,

$$
\left|\left[\left(D^{\beta} K_{\delta}\right) * f\right]^{\wedge}(\xi)\right| \leqslant C|\hat{f}(\xi)| .
$$

The lemma now follows from the isometry of the transform on $H^{0}$.

To prove Lemma 3.8, we need the following fact:

LEMmA 5.2. Given $f \in H^{-1}\left(\mathbf{R}^{3} ; \mathbf{R}\right)$ with supp $f \subseteq\{|z|<R\}$, there exist $f_{0} \in$ $H^{0}\left(\mathbf{R}^{3} ; \mathbf{R}\right)$ and $f_{1} \in H^{0}\left(\mathbf{R}^{3} ; \mathbf{R}^{3}\right)$, both with support in $\{|z|<2 R\}$, so that

$$
f=f_{0}+\nabla \cdot f_{1}, \quad\left|f_{0}\right|_{0}+\left|f_{1}\right|_{0} \leqslant C|f|_{-1} .
$$

Proof. Using a cutoff function, we write $\hat{f}(\xi)=\gamma_{0}(\xi)+\gamma_{1}(\xi)$ with $\gamma_{0}$ of bounded support and $\gamma_{1}=0$ in a neighborhood of $\xi=0$. Then

$$
\gamma_{1}=2 \pi i \xi \cdot\left((2 \pi i)^{-1} \xi|\xi|^{-2} \gamma_{1}\right) \equiv 2 \pi i \xi \cdot \tilde{\gamma}_{1}
$$

Define $g_{0}, g_{1}$ by $\hat{g}_{0}=\gamma_{0}, \hat{g}_{1}=\tilde{\gamma}_{1}$. We now have $f=g_{0}+\nabla \cdot g_{1}$, with an inequality like (5.15) satisfied, but we still have to restrict the support. Let $\phi$ be a cutoff function with $\phi=1$ for $|z| \leqslant R, \phi=0$ for $|z| \geqslant 2 R$. Then

$$
f=\phi f=\phi g_{0}+\phi\left(\nabla \cdot g_{1}\right)=\left(\phi g_{0}-\nabla \phi \cdot g_{1}\right)+\nabla \cdot\left(\phi g_{1}\right) \text {. }
$$

The choice

$$
f_{0}=\phi g_{0}-\nabla \phi \cdot g_{1}, \quad f_{1}=\phi g_{1}
$$

meets our requirements. 
Proof of Lemma 3.8. Given $f \in H^{-1}\left(\mathbf{R}^{3} ; \mathbf{R}^{3}\right)$, we can apply Lemma 5.2 to each component of $f$ and write $f=\Sigma_{|\beta| \leqslant 1} D^{\beta} f_{\beta}$ with $\left|f_{\beta}\right|_{0} \leqslant C|f|_{-1}$ and supp $f_{\beta} \subseteq\{|z|<$ $2 R\}$. Now $K_{\delta} *\left(D^{\beta} f_{\beta}\right)=\left(D^{\beta} K_{\delta}\right) * f_{\beta}$, and from Lemma 3.7 we have for $|\beta|=1$

$$
\left|K_{\delta} *\left(D^{\beta} f_{\beta}\right)\right|_{0} \leqslant C\left|f_{\beta}\right|_{0} \leqslant C^{\prime}|f|_{-1} \text {. }
$$

Therefore we need only show that

$$
\left|K_{\delta} * f_{0}\right|_{H^{0}(|z|<R)} \leqslant C\left|f_{0}\right|_{H^{0}(|z|<2 R)} .
$$

According to Lemma 3.1, this inequality holds provided

$$
\int_{|z|<3 R}\left|K_{\delta}(z)\right| d z \leqslant C
$$

and this in turn follows from Lemma 3.3.

Department of Mathematics

Tulane University

New Orleans, Louisiana 70118

Department of Mathematics

University of California, Berkeley

Berkeley, California 94720

I. J. T. Beale \& A. Majda, "Vortex methods. II: Higher order accuracy in two and three dimensions," Math. Comp., v. 39, 1982, pp. 29-52.

2. J. T. Beale \& A. Majda, The Design and Analysis of Vortex Methods, Proc. Conf. on Transonic, Shock, and Multi-Dimensional Flows, Madison, Wisc., May 1981. (To appear.)

3. A. J. ChORIn, “Numerical study of slightly viscous flow,” J. Fluid Mech., v. 57, 1973, pp. 785-796.

4. A. J. ChORIN, "Vortex models and boundary layer instability," SIAM J. Sci. Statist. Comput., v. 1, 1980, pp. 1-21.

5. A. J. Chorin, "Estimates of intermittency, spectra, and blow-up in developed turbulence," Comm. Pure Appl. Math. (To appear.)

6. A. J. Chorin \& P. Bernard, "Discretization of vortex sheet with an example of roll-up," J. Comput. Phys., v. 13, 1973, pp. 423-428.

7. A. J. Chorin \& J. MARSDen, A Mathematical Introduction to Fluid Mechanics, Springer-Verlag, New York, 1979.

8. V. Del Prete, Numerical Simulation of Vortex Breakdown, L.B.L. Math. and Comp. Report, 1978.

9. G. Folland, Introduction to Partial Differential Equations, Princeton Univ. Press, Princeton, N. J., 1978.

10. O. Hald, "The convergence of vortex methods, II," SIAM J. Numer. Anal., v. 16, 1979, pp. $726-755$.

11. O. Hald, "Convergence of Fourier methods for the Navier-Stokes equations," J. Comput. Phys. (To appear.)

12. J. K. Hale, Ordinary Differential Equations, Wiley-Interscience, New York, 1969.

13. T. Kato, "Nonstationary flows of viscous and ideal fluids in $\mathrm{R}^{3}$, , J. Funct. Anal., v. 9, 1972, pp. 296-305.

14. H. O. Kreiss \& J. Oliger, "Comparison of accurate methods for the integration of hyperbolic equations," Tellus, v. 24, 1972, pp. 199-215.

15. H. O. Kreiss \& J. Oliger, "Stability of the Fourier method," SIAM J. Numer. Anal., v. 16, 1979. pp. 421-433.

16. A. Leonard, “Vortex methods for flow simulations,” J. Comput. Phys., v. 37, 1980, pp. 289-335.

17. A. LeONARD, Numerical Simulation of Interacting Three-Dimensional Vortex Filaments, Proc. 4th Internat. Conf. Numer. Methods Fluid Dynamics, Springer-Verlag, New York, 1975, pp. 245-249.

18. A. LeONARD, Simulation of Three-Dimensional Separated Flow's with Vortex Filaments, Proc. 5th Internat. Conf. Numer. Methods Fluid Dynamics, Springer-Verlag, New York, 1977, pp. 280-284.

19. A. Majda, J. McDonough \& S. Osher, "The Fourier method for nonsmooth initial data," Math. Comp., v. 32, 1978, pp. 1041-1081. 
20. L. Rosenhead, "The point vortex approximation of a vortex sheet," Proc. Roy. Soc. London Ser. A, v. 134, 1932, pp. 170-192.

21. E. Stein \& G. Weiss, Fourier Analysis on Euclidean Space, Princeton Univ. Press, Princeton, N. J., 1971.

22. R. Temam, "Local existence of $C^{\infty}$ solutions of the Euler equations of incompressible perfect fluids," in Turbulence and the Navier-Stokes Equations, Springer-Verlag, New York, 1976, pp. 184-194.

23. R. Temam, The Navier-Stokes Equations, North-Holland, Amsterdam, 1977.

24. K. KuWAHARA \& H. TAKAMI, "Numerical studies of two-dimensional vortex motion by a system of point vortices," J. Phys. Soc. Japan, v. 34, 1973, pp. 247-253.

25. L. M. MiLNe-ThOMPson, Theoretical Hydrodynamics, 4th ed., Macmillan, New York, 1960.

26. J. Serrin, Mathematical Principles of Classical Fluid Mechanics, Handbuch der Physik VIII/I, Springer-Verlag, Berlin, 1959. 